\title{
ARITHMETICS AND COMBINATORICS OF TROPICAL SEVERI VARIETIES OF UNIVARIATE POLYNOMIALS
}

\author{
ALICIA DICKENSTEIN, MARÍA ISABEL HERRERO, LUIS FELIPE TABERA
}

\begin{abstract}
We give a description of the tropical Severi variety of univariate polynomials of degree $n$ having two double roots. We show that as a set, it is given as the union of three explicit types of cones of maximal dimension $n-1$, where only cones of two of these types are cones of the secondary fan of $\{0, \ldots, n\}$. Through Kapranov's theorem, this goal is achieved by a careful study of the possible valuations of the elementary symmetric functions of the roots of a polynomial with two double roots. Despite its apparent simplicity, the computation of the tropical Severi variety has both combinatorial and arithmetic ingredients.
\end{abstract}

\section{INTRODUCTION}

Moduli spaces of various objects are of primary interest in algebraic geometry. Among them, Severi varieties are classical objects which give parameter spaces for nodal hypersurfaces. Starting with [12, techniques from tropical geometry have been interestingly applied to the study of classical enumerative problems. Mikhalkin's correspondence theorem allows to compute tropically the number of planar curves of degree $d$ and any number $\delta$ of nodes passing through $\frac{(d+3) d}{2}-\delta$ points in general position, that is, the degree of the Severi variety parametrizing those curves or, more generally, the degree of the Severi varieties of nodal curves with $\delta$ nodes defined by polynomials with support in (the lattice points of) a given lattice polygon. For curves of degree $d \geq \delta+2$, the degree of the Severi variety coincides with the Gromov-Witten invariant $N_{d,\left(\begin{array}{c}d-1 \\ 2\end{array}\right)-\delta}$. We refer the reader to [2] for an introduction to tropical geometry techniques for algebraic curve counting problems.

Consider a (finite) lattice configuration $A$ in any dimension (for instance, all the lattice points in the $d$-th dilate of a unit simplex). Order the points in $A$ and denote by $\mathcal{A}$ the matrix having these points as columns. The secondary fan of $A$ (defined and studied in [9]) parametrizes the regular polyhedral subdivisions of the configuration. In the case of a single node, the Severi variety corresponding to polynomials with support in $A$ is defined by the $A$-discriminant. It was proven in 5 that the tropical $A$-discriminant coincides with the Minkowski sum of the row span of $\mathcal{A}$ and the tropicalization of the kernel of $\mathcal{A}$, and it is the union of some cones in the secondary fan of $A$. Severi varieties of polynomials with support in $A$ and increasing number of nodes form a natural stratification of the $A$-discriminant.

As a consequence of the general position of the points, the tropical curves appearing in Mikhalkin's correspondence theorem can be described by the associated regular subdivision of the support. That is, the set of tropical curves with a specified combinatorial type counted in Mikhalkin's formula correspond to polyhedral cones in the associated secondary fan. However, these cones are a fraction of all possible cones in the associated tropical Severi variety. E. Katz noted in 10 that there are maximal cones that are not supported in cones of the secondary fan of $A$. Thus, the combinatorial description of the curves is not enough in many cases 
to decide if a tropical curve given by a tropical polynomial is in the corresponding Severi variety or not. This behavior was also observed by J. J. Yang, who gave a partial description of the tropicalization of the Severi varieties in [17, 18. We explore this phenomenon and give a full characterization in the univariate setting for the case of $\delta=2$ nodes. Besides the combinatorial constraints, we describe arithmetic restrictions.

A recent interesting paper of Esterov [8] defines affine characteristic classes of affine varieties, which govern equivariant enumerative problems. The computation of the fundamental class of a hypersurface of the torus in the corresponding affine cohomology ring, amounts to the computation of the Newton polytope of a defining equation. In general, for a subvariety of the torus of any codimension, the computation of its fundamental class amounts to the computation of its tropical fan. The codimension two strata of the $A$-discriminant are given by the closure of the hypersurfaces with support in $A$ with one triple root or those hypersurfaces with two nodes, that is, the 2 -Severi variety. Esterov gives affine Plücker formulas relating these classes with the class of the $A$-discriminant.

Our setting is the following. We fix a natural number $n \geq 4$. The Severi variety $S e v_{n}^{2}$ is the Zariski closure of the set of univariate polynomials of degree $n$ having 2 distinct double roots (over an algebraically closed field of characteristic zero). $S e v_{n}^{2}$ is a rational variety (with a rational parametrization defined over $\mathbb{Q}$ ) and thus irreducible, of affine dimension $n-1$. The tropical Severi variety $\mathcal{T}\left(\operatorname{Sev}_{n}^{2}\right)$ is the tropicalization of $S e v_{n}^{2}$. Despite its apparent simplicity, the computation of the tropicalization $\mathcal{T}\left(S e v_{n}^{2}\right)$ has both combinatorial and arithmetic ingredients.

We describe in Section 3 three types of cones with maximal dimension $n-1=$ $n+1-2$, termed I, II and III. Cones of type I correspond to two double roots with different valuations and arise from the transversal intersection of two maximal dimensional cones in the discriminant. Cones of types II and III correspond to roots which are not tropically generic: both roots need to have the same valuation, and the relative interior of these cones correspond to the tropicalization of polynomials with two nodes whose initial coefficients satisfy explicit algebraic relations. Moreover, cones of type III intersect the interior of a maximal cone in the secondary fan of our configuration $\{0,1, \ldots, n\}$ of exponents. The existence of a second node imposes a "hidden tie" with arithmetic constraints.
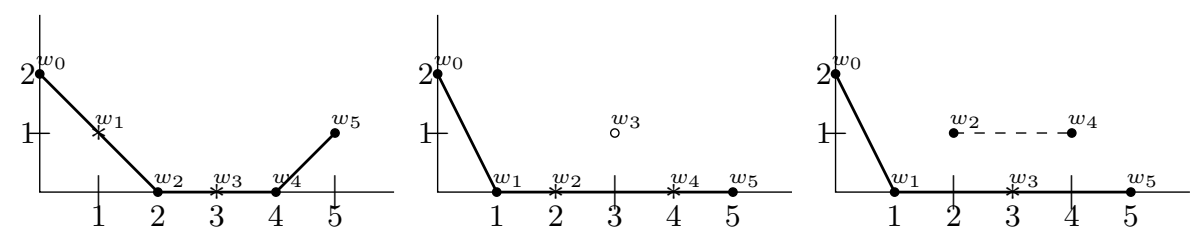

Figure 1. Left: Type I, Center: Type II, Right: Type III

To explain Figure 1 featuring the three types of cones in $\mathcal{T}\left(S e v_{n}^{2}\right)$, we need to set some notations. Any vector $w \in \mathbb{R}^{n+1}$ defines a marked subdivision $\Pi_{w}$ of $\mathcal{A}=\{0, \ldots, n\}$ as follows. Let $\Gamma_{1}, \ldots, \Gamma_{s}$ be the lower faces of the convex hull of the set $\Gamma_{w}=\left\{\left(0, w_{0}\right), \ldots,\left(n, w_{n}\right)\right\}$, known as the Newton diagram of $w$. Let $\sigma_{1}, \ldots, \sigma_{s}$ be the subsets of $\mathcal{A}$ obtained by projecting the points of $\Gamma_{w}$ in each face $\Gamma_{1}, \ldots, \Gamma_{s}$ onto the first coordinate. These subsets (or cells) determine a regular subdivision $\Pi_{w}=\left\{\sigma_{1}, \ldots, \sigma_{s}\right\}$ of $\mathcal{A}$. A point $j$ is a marked point in the cell $\sigma_{i}$ if $\left(j, \operatorname{val}\left(w_{j}\right)\right)$ is a point in the relative interior of the face $\Gamma_{i}$. In that case, the cell $\sigma_{i}$ is called a marked cell. Figure 1 depicts for $n=5$, the Newton diagrams corresponding to weights $w \in \mathbb{R}^{6}$ in the interior of one sample cone of each type. Note that 
for points $w$ in (the relative interior of) cones of type I, there are two different marked segments (with one marking each) in $\Pi_{w}$. For points $w$ in (the relative interior of) cones of type II, there is a single segment in $\Pi_{w}$ with two markings (and the exceptional small configurations in Definition 3.5 should be excluded by Proposition 4.6); these are also cones that arise from the intersection of two maximal dimensional cones of the tropical discriminant. Regular subdivisions $\Pi_{w}$ associated with points $w$ in (the relative interior of) cones of type III, have a single marked segment with a single marking, but there is also a tie between values that do not lie in the Newton diagram, that is, which is not visible from the marked subdivision (thus called a "hidden tie"). Moreover, we will see in Definition 3.8 that there are arithmetic ingredients which control these values. These cones arise from two nodes with the same valuation and with further algebraic relations on the initial coefficients. They don't have for the moment a purely tropical explanation. We refer to Definitions 3.3, 3.6 and 3.8 for the precise conditions.

Our main result is the following:

Theorem 1.1. Let $n \geq 4$. Then, $\mathcal{T}\left(S e v_{n}^{2}\right) \subset \mathbb{R}^{n+1}$ equals as a set the union of all cones of types $I, I I$ and $I I I$.

The proof of Theorem 1.1 follows from a series of results. On one side, Theorem 4.8 shows that $\mathcal{T}\left(S e v_{n}^{2}\right)$ is contained in the union of the cones of types $I, I I$ and $I I I$. On the other side, Theorem 5.1 proves that cones of type I lie in $\mathcal{T}\left(S e v_{n}^{2}\right)$, Theorems 5.3 and 5.4 show that cones of type II lie in $\mathcal{T}\left(S e v_{n}^{2}\right)$, and finally, Theorem 5.5 gives the last inclusion of cones of type III in the tropicalization of the Severi variety.

The article is structured as follows, In Section 2 we present some general results concerning the tropicalization of homogeneous linear ideals over an infinite valuated ring and we give a characterization using maximal minors of an associated matrix in Theorem 2.6. In Section 3, some known results and notation are stated and a detailed description of cones of type $I, I I$ and $I I I$ is given. We prove in Section 4 that $\mathcal{T}\left(S e v_{n}^{2}\right)$ is contained in the union of the cones of type $I, I I$ and $I I I$, while Section 5 deals with the converse inclusion. Finally, we include three separated appendices for the convenience to the reader. The first one contains a technical result about linear spaces and circuits. The second one contains a set of results about minors of the matrices that appear naturally when studying $S e v_{n}^{2}$. The last Appendix contains code that has been used to verify some claims along the text.

Note that our approach in Section 2 is very general (independent of $n$ and $\delta$ ) and could be used to describe the case of any number $\delta$ of nodes. However, we restricted our attention to the particular case $\delta=2$, which highlights the new phenomena that occur in type III cones and that already requires many technical results. Our approach is used in Example 2.7 to clarify the two dimensional case worked out in $[8]$.

\section{RESUlts ON HOMOGENEOUS LINEAR IDEALS}

In this section we present some basic results about the tropicalization of linear homogeneous ideals over a valuated field. We refer the reader to the book [1] for basic results on valuations and tropicalizations.

Along the paper, $\mathbb{K}$ will denote an algebraically closed field with a rank one non-archimedean valuation

$$
\text { val }: \mathbb{K}^{*} \rightarrow \mathbb{R}
$$

such that both $\mathbb{K}$ and its residue field $K$ are of characteristic zero. This is equivalent to the fact that integers have valuation 0 . The value group $\Gamma$ is dense in $\mathbb{R}$ and possibly multiplying val by a constant, we may assume that $\Gamma$ contains the 
rationals. As $\mathbb{K}$ is algebraically closed, there exists a split of the valuation. That is, a multiplicative subgroup $\left\{t^{a}: a \in \operatorname{val}\left(\mathbb{K}^{*}\right)\right\} \subseteq \mathbb{K}^{*}$ isomorphic to $\Gamma$.

If $b \in \mathbb{K}^{*}$ with $\operatorname{val}(b)=v$, we will usually write $b=\beta t^{v}+* \in \mathbb{K}, \beta \in K^{*}$, to distinguish the lowest term. The reader may assume for simplicity that $\mathbb{K}=\mathbb{C}\{\{t\}\}$, the field of Puiseux series with its usual valuation, and $K=\mathbb{C}$.

Given a polynomial $g=\sum_{\alpha \in \mathbb{N}^{n+1}} g_{\alpha} x^{\alpha} \in \mathbb{K}\left[x_{0}, \ldots, x_{n}\right]$ (or in the Laurent polynomial ring $\left.\mathbb{K}\left[x_{0}^{ \pm 1}, \ldots, x_{n}^{ \pm 1}\right]\right)$, the tropicalization $\operatorname{trop}(g)$ of $g$ is the piecewise linear function on $\mathbb{R}^{n+1}$ defined by:

$$
\operatorname{trop}(g)(w)=\min \left\{\operatorname{val}\left(g_{\alpha}\right)+\langle w, \alpha\rangle: g_{\alpha} \neq 0\right\},
$$

and its zero set $V(\operatorname{trop}(g)) \subset \mathbb{R}^{n+1}$ is given by those $w$ for which the minimum in $\operatorname{trop}(g)$ is attained at least twice. The tropicalization $\mathcal{T}(I)$ of an ideal $I$ in the Laurent polynomial ring is defined as the intersection of the zero sets $V(\operatorname{trop}(g))$ for any nonzero $g \in I$. The tropicalization $\mathcal{T}(X)$ of an algebraic subvariety $X$ of the torus is the tropicalization of its ideal. Given an affine or projective irreducible variety, its tropicalization is defined as the tropicalization of its intersection with the torus. In fact, for any ideal $I$, its tropicalization $\mathcal{T}(I)$ can be given as a finite intersection, and any finite subset of polynomials in $I$ which suffice to describe $\mathcal{T}(I)$ is called a tropical basis of $I([3])$.

Lemma 2.2 below tells us that any linear homogeneous ideal has a tropical basis formed by circuits, as in the well known case of a trivial valuation. For the convenience of the reader, we include in the Appendix a Gröbner free proof, which is adapted from Lemma 3.12 and Theorem 3.13 in [14.

We first recall the definition of circuits.

Definition 2.1. Let $I$ be an homogeneous linear ideal in $\mathbb{K}\left[x_{0}, \ldots, x_{n}\right]$. A circuit in $I$ is a non-zero linear form $\ell \in I$ with minimal support. Let $M$ be a matrix in $\mathbb{K}^{d \times(n+1)}$. A circuit in $M$ is a non-zero element $r \in \operatorname{rowspan}(M)$ with minimal support.

Note that there is a finite number of circuits in $I$ (up to a multiplicative constant). Clearly, if we interpret the rows of a matrix $M$ as coefficients of linear forms in variables $x_{0}, \ldots, x_{n}$, the circuits of $M$ coincide with the circuits of the ideal $I(M)$ generated by these $d$ linear forms.

Lemma 2.2. Let $I \subset \mathbb{K}\left[x_{0}, \ldots, x_{n}\right]$ be a homogeneous linear ideal. Then

$$
\mathcal{T}(I)=\bigcap_{\substack{\ell \in I \\ \ell \text { circuit }}} V(\operatorname{trop}(\ell)) .
$$

Remark 2.3. Lemma 2.2 holds under the weaker hypotheses that $\mathbb{K}$ is a valuated field (not necessarily algebraically closed) with a rank one valuation such that the residue field is infinite. In particular, Theorem 2.6 below holds in the positive characteristic case and the $p$-adic case.

The hypothesis that the residue field is infinite cannot be avoided. Let $\mathbb{F}_{3}$ be the field of three elements and consider $\mathbb{K}=\mathbb{F}_{3}(t)$ with the usual valuation. Let $I$ be the linear ideal generated by $x_{1}-x_{4}+x_{6}, x_{2}-x_{4}-x_{6}, x_{3}+x_{4}, x_{5}+x_{6}$. Then $(0,0,0,0,0,0) \in \operatorname{trop}(\ell)$, for every linear form $\ell \in I$. However, there is no element in $V(I)$ with coefficients in $\mathbb{F}_{3}(t)$ whose tropicalization is $(0,0,0,0,0,0)$. On the other hand, in $\overline{\mathbb{F}_{3}}(t)$, we have that the point $(2+\alpha, 1+\alpha, 2 \alpha, \alpha, 2,1) \in V(I)$, with $\alpha^{2}+1=0$. Its tropicalization is the desired point $(0,0,0,0,0,0)$.

Now we want to characterize the circuits of a matrix $M \in \mathbb{K}^{d \times(n+1)}$ in terms of its minors. 
Notation 2.4. Let $M$ be a matrix in $\mathbb{K}^{d \times(n+1)}$. Let $J=\left\{i_{1}, \ldots, i_{s}\right\} \subseteq\{0, \ldots, n\}$ with $i_{1}<\cdots<i_{s}$. If we denote $C_{0}, \ldots, C_{n}$ the ordered columns of the matrix $M$, then $M_{J}$ is defined as the matrix in $\mathbb{K}^{d \times s}$ with columns $C_{i_{1}}, \ldots, C_{i_{s}}$. If $s=d-1$, then $r_{J} \in \operatorname{rowspan}(M)$ is the vector in $\mathbb{K}^{(n+1)}$ defined by $r_{J, k}=$ $(-1)^{\mu(k, J)} \operatorname{det}\left(M_{J \cup\{k\}}\right)$, where $\mu(k, J)$ is the sign of the permutation of $J \cup\{k\}$ which takes $k$ followed by the ordered elements of $J$ to the ordered elements of $J \cup\{k\}$, for all $k \in\{0, \ldots, n\}$.

Without loss of generality, $M$ can be assumed to be of maximal rank. The following lemma is straightforward

Lemma 2.5. Let $M \in \mathbb{K}^{d \times(n+1)}$ be a matrix of rank $d$ and $J \subset\{0, \ldots, n\}$ such that $\# J=d-1$ and $\operatorname{rank}\left(M_{J}\right)=d-1$. Then $r_{J} \in \mathbb{K}^{(n+1)}$ is a circuit of $M$. Moreover, up to multiplicative constant, these are all the circuits of $M$ (possibly repeated).

We can now describe precisely the tropicalization of the kernel of $M$.

Theorem 2.6. Let $M \in \mathbb{K}^{d \times(n+1)}$ be a matrix of rank $d$ and $w \in \mathbb{R}^{n+1}$. Then $w \in \mathcal{T}(\operatorname{ker}(M))$ if and only if, for every $J \subseteq\{0, \ldots, n\}$ with $d-1$ elements such that $\operatorname{rank}\left(M_{J}\right)=d-1$, the minimum of

$$
\left\{\operatorname{val}\left(\operatorname{det}\left(M_{J \cup\{k\}}\right)\right)+w_{k} \quad \mid \quad k \in\{0, \ldots, n\}\right\}
$$

is attained at least twice.

Proof. Given $M$, let $I(M)$ be the homogeneous linear ideal generated by the linear forms associated with the elements in $\operatorname{rowspan}(M)$. For every linear form $\ell(x)=$ $\sum_{i=0}^{n} \ell_{i} x_{i}$ in $I(M), w \in V(\operatorname{trop}(\ell))$ if and only if the initial form

$$
\sum_{\substack{\operatorname{val}\left(\ell_{i}\right)+w_{i} \\ \text { minimal }}} \ell_{i} x_{i}
$$

is not a monomial, which is equivalent to $\min \left\{\operatorname{val}\left(\ell_{0}\right)+w_{0}, \ldots, \operatorname{val}\left(\ell_{n}\right)+w_{n}\right\}$ being attained at least twice.

Because of the Lemma 2.2. $\mathcal{T}(\operatorname{ker}(M))=\mathcal{T}(I(M))=\bigcap V(\operatorname{trop}(\ell))$ where the intersection is over all circuits in $I(M)$. Then, $w \in \mathcal{T}(\operatorname{ker}(M))$ if and only if for every circuit $r=\left(r_{0}, \ldots, r_{n}\right) \in \operatorname{rowspan}(M)$, there are at least two elements in the set $\left\{i \in\{0, \ldots, n\}: \operatorname{val}\left(r_{i}\right)+w_{i}=\min \left\{\operatorname{val}\left(r_{0}\right)+w_{0}, \ldots, \operatorname{val}\left(r_{n}\right)+w_{n}\right\}\right\}$. The result follows from Lemma 2.5 and the fact that $\operatorname{val}\left(-r_{i}\right)=\operatorname{val}\left(r_{i}\right)$.

As an example, we use Theorem 2.6 to present a new explanation of the cones in the tropicalization of the set of bivariate polynomials with two nodes given in Example 3.9 of 8 .

Example 2.7. Consider the set $\mathrm{A}=\{(0,0),(1,0),(1,1),(0,1),(-1,0),(0,-1)\}$ and the variety $S e v_{\mathrm{A}}^{2}$, given as the (Zariski) closure of the set

$\left\{c \in\left(\mathbb{K}^{*}\right)^{6}: f(x, y)=c_{1}+c_{2} x+c_{3} x y+c_{4} y+c_{5} \frac{1}{x}+c_{6} \frac{1}{y}\right.$ has two nodes in $\left.\left(\mathbb{K}^{*}\right)^{2}\right\}$.

Consider the subvariety $S \subset S e v_{\mathrm{A}}^{2}$ given by the closure of those $c=\left(c_{1}, \ldots, c_{6}\right)$ with a node at the fixed point $(1,1)$ plus another node that we call $\left(b_{1}, b_{2}\right) \in$ $\left(\mathbb{K}^{*}\right)^{2} \backslash\{(1,1)\}$. Then, such a $c$ satisfies $c \in \operatorname{ker}\left(M^{\prime}\right)$, where

$$
M^{\prime}=\left(\begin{array}{ccccrr}
1 & 1 & 1 & 1 & 1 & 1 \\
0 & 1 & 1 & 0 & -1 & 0 \\
0 & 0 & 1 & 1 & 0 & -1 \\
b_{1} b_{2} & b_{1}^{2} b_{2} & b_{1}^{2} b_{2}^{2} & b_{1} b_{2}^{2} & b_{2} & b_{1} \\
0 & b_{1}^{2} & b_{1}^{2} b_{2} & 0 & -1 & 0 \\
0 & 0 & b_{1} b_{2}^{2} & b_{2}^{2} & 0 & -1
\end{array}\right) .
$$


As $\operatorname{det}\left(M^{\prime}\right)=b_{1} b_{2}\left(b_{1}-1\right)^{2}\left(b_{2}-1\right)^{2}\left(b_{1} b_{2}-1\right), M^{\prime}$ has a non-trivial kernel if and only if $b_{1}=1$ or $b_{2}=1$ or $b_{2}=\frac{1}{b_{1}}$. In these cases, it can be checked that $\operatorname{rank}\left(M^{\prime}\right)=5$.

We will call $M \in \mathbb{K}^{5 \times 6}$ the submatrix of $M^{\prime}$ of maximal rank obtained by removing the 5 -th row if $\left(b_{1}, b_{2}\right) \in\{(-1,1),(-1,-1)\}$, the 6 th row if $\left(b_{1}, b_{2}\right)=$ $(1,-1)$, and the 4 -th row for very other $\left(b_{1}, b_{2}\right)$ where the determinant vanishes. Applying Theorem 2.6 we have that $w \in \mathcal{T}(\operatorname{ker}(M))$ if and only if, for every $J \subseteq\{1, \ldots, 6\}$ with 4 elements and $\operatorname{rank}\left(M_{J}\right)=4$, the minimum of

$$
\left\{\operatorname{val}\left(\operatorname{det}\left(M_{J \cup\{k\}}\right)\right)+w_{k} \quad \mid \quad k \in\{1, \ldots, 6\} \backslash J\right\}
$$

is attained at least twice. Because of the size of this example, this can be translated into de fact that for both $k_{1}, k_{2} \in\{1, \ldots, 6\} \backslash J, \operatorname{val}\left(\operatorname{det}\left(M_{J \cup\left\{k_{1}\right\}}\right)\right)+w_{k_{1}}=$ $\operatorname{val}\left(\operatorname{det}\left(M_{J \cup\left\{k_{2}\right\}}\right)\right)+w_{k_{2}}$. Based on this theorem, we can easily discard $b_{1}=1$ or $b_{2}=1$. For instance, if $b_{2}=1$, taking $J=\{1,2,3,4\}$ we obtain that the minimum in Equation (2) is only attained once at $k=6$.

We now describe all the cones in $\mathcal{T}\left(S e v_{\mathrm{A}}^{2}\right)$ when $b_{2}=\frac{1}{b_{1}}$. As before, the case $\left(b_{1}, b_{2}\right)=(-1,-1)$ can be discarded by taking the set $J=\{2,3,4,5\}$ for which we obtain that the minimum in Equation (2) is only attained once at $k=1$.

For every $\left(b_{1}, b_{2}\right) \in\left(\mathbb{K}^{*}\right)^{2}-\{(-1,-1),(1,1)\}$, let $M_{i}$ be the $5 \times 5$ matrix obtained by removing the $i$-th column from $M$. Then, we can compute

$$
\begin{gathered}
\operatorname{det}\left(M_{1}\right)=-\operatorname{det}\left(M_{3}\right)=\frac{\left(b_{1}-1\right)^{2}\left(b_{1}+1\right)^{2}}{b_{1}^{2}}, \operatorname{det}\left(M_{4}\right)=\operatorname{det}\left(M_{5}\right)=-\frac{\left(b_{1}-1\right)^{2}\left(b_{1}+1\right)}{b_{1}} \\
\text { and } \operatorname{det}\left(M_{2}\right)=-\operatorname{det}\left(M_{6}\right)=-\frac{\left(b_{1}-1\right)^{2}\left(b_{1}+1\right)}{b_{1}^{2}} .
\end{gathered}
$$

Since none of them vanishes at $\left(b_{1}, b_{2}\right)$, every $J \subset\{1,2,3,4,5,6\}$ with 4 elements satisfies that $\operatorname{rank}\left(M_{J}\right)=4$.

When $\operatorname{val}\left(b_{1}\right) \neq 0$, using Theorem 2.6 and considering the valuations of the determinants of the matrices $M_{i}$, we obtain that $w \in \mathcal{T}(\operatorname{ker}(M))$ if and only if one of the following sets of equations is verified

$$
\begin{aligned}
& w_{1}=w_{2}=w_{3}=w_{6}<w_{4}=w_{5} \text { if } \operatorname{val}\left(b_{1}\right)>0 \\
& w_{1}=w_{3}=w_{4}=w_{5}<w_{2}=w_{6} \text { if } \operatorname{val}\left(b_{1}\right)<0 .
\end{aligned}
$$

These points induce the subdivision of the convex hull of A in Figure 2 .

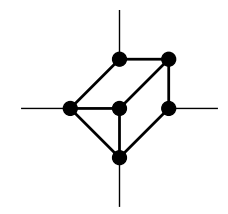

Figure 2. $\operatorname{val}\left(b_{1}\right) \neq 0$

When $\operatorname{val}\left(b_{1}\right)=0$ and $b_{1}=\beta+*$ where $\beta \neq-1$, we obtain points in $\langle(1,1,1,1,1,1)\rangle$.

When $\operatorname{val}\left(b_{1}\right)=0$ and $b_{1}=-1+*\left(b_{1} \neq-1\right)$, we obtain points $w \in\left(\mathbb{K}^{*}\right)^{6}$ that induce the regular subdivision in Figure 3 .

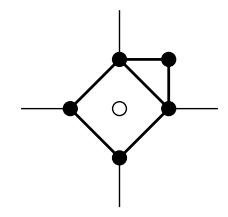

Figure 3. $\operatorname{val}\left(b_{1}\right)=0, \beta=-1$ 
This subdivision seems to be associated with the existence of one node, but the existence of a second node imposes a "hidden tie" from the relations

$$
w_{1}=w_{3}>w_{2}=w_{4}=w_{5}=w_{6} .
$$

Thus, we get the subdivision that Esterov represents with the dotted segment representing the hidden tie depicted in Figure 4.

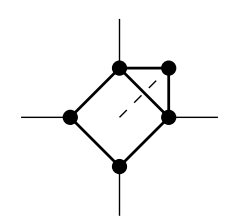

Figure 4. Hidden tie

Indeed, $\mathcal{T}(S)$ is a tropical linear space of dimension two. Now, we add the action of the linear space rowspan $(A)$, where $A$ is the $2 \times 6$ matrix of exponents of our configuration read in the second and third rows of $M^{\prime}$ to get $\mathcal{T}\left(S e v_{\mathrm{A}}^{2}\right)$ as the union of the two cones giving the subdivisions and hidden tie presented in Example 3.9 of [8]. These cones are explicitly $\mathcal{C}_{\diamond}$, with $\diamond$ equal to $\leq$ and to $\geq$ :

$$
\mathcal{C}_{\diamond}=\left\{w \in \mathbb{R}^{6}: w_{1}-w_{3}=w_{5}-w_{4}=w_{6}-w_{2}, 4 w_{1} \diamond w_{2}+w_{4}+w_{5}+w_{6}\right\} .
$$

In fact, $S e v_{\mathrm{A}}^{2}$ is in this case a toric variety, defined by the equations

$$
c_{2} c_{5}-c_{4} c_{6}=0, c_{1} c_{4}-c_{3} c_{5}=0, c_{1} c_{2}-c_{3} c_{6}=0 .
$$

\section{DEFINING THE CONES}

In this section, we describe the cones in $\mathcal{T}\left(S e v_{n}^{2}\right)$.

3.1. Notation and basic facts. Let $K$ be an algebraically closed field of characteristic zero, $n \in \mathbb{N}_{\geq 4}$, and let $\mathcal{U} \subset K^{n-1}$ be the open set defined by

$$
\mathcal{U}=\left\{\left(a, b, a_{1}, \ldots, a_{n-3}\right) \in\left(K^{*}\right)^{n-1}: a, b, a_{1}, \ldots, a_{n-4} \text { are all different }\right\} .
$$

The Severi variety $\operatorname{Sev}_{n}^{2}(K)$ is the Zariski closure of the image of the map $\varphi_{K}$ defined by:

$$
\begin{aligned}
\mathcal{U} & \longrightarrow K^{n+1} \\
\left(a_{1}, a_{2}, a_{3}, \ldots, a_{n-1}\right) & \mapsto\left(c_{0}, \ldots, c_{n}\right),
\end{aligned}
$$

where $c=\left(c_{0}, \ldots, c_{n}\right)$ denotes the vector of coefficients of the polynomial

$$
f=\sum_{i=0}^{n} c_{i} x^{i}=a_{n-1}\left(x-a_{1}\right)^{2}\left(x-a_{2}\right)^{2} \prod_{i=3}^{n-2}\left(x-a_{i}\right) .
$$

Thus, $I\left(\operatorname{Sev}_{n}^{2}(K)\right)$ is defined over $\mathbb{Q}$.

Let $\mathbb{K}$ be an algebraically closed valuated extension of $K$, with residue field $K$. For any $c$ in the torus $\left(\mathbb{K}^{*}\right)^{n+1}$, we denote by $\operatorname{val}(c)=\left(\operatorname{val}\left(c_{0}\right), \ldots, \operatorname{val}\left(c_{n}\right)\right)$ and we will identify $c$ with the univariate polynomial $f$ with coefficients $c$. The image $\operatorname{val}\left(\left(\mathbb{K}^{*}\right)^{n+1}\right) \subset \mathbb{R}^{n+1}$ will be denoted by $\operatorname{Im}_{\text {val }}$. Also, for any $w \in \operatorname{Im}_{\text {val }}$ we denote by $\mathbb{K}[x]_{w}$ the set of polynomials $f$ with $w=\operatorname{val}(c)$.

By Theorems 3.2.2 and 3.2.4 in [1],

$$
\mathcal{T}\left(\operatorname{Sev}_{n}^{2}(K)\right)=\mathcal{T}\left(\operatorname{Sev}_{n}^{2}(\mathbb{K})\right)=\operatorname{closure}\left\{\operatorname{val}(c), c \in \operatorname{Im}\left(\varphi_{\mathbb{K}}\right) \cap\left(\mathbb{K}^{*}\right)^{n+1}\right\} .
$$

As the field is not essential, we will denote in what follows the tropicalization of our Severi varieties by $\mathcal{T}\left(S e v_{n}^{2}\right)$. 
Definition 3.1. Let $\mathbf{M} \in(\mathbb{Z}[x])^{4 \times(n+1)}$ be the matrix

$$
\mathbf{M}=\left(\begin{array}{ccccc}
1 & 1 & 1 & \ldots & 1 \\
0 & 1 & 2 & \ldots & n \\
1 & x & x^{2} & \ldots & x^{n} \\
0 & x & 2 x^{2} & \ldots & n x^{n}
\end{array}\right)
$$

Let $J=\left\{i_{1}, i_{2}, i_{3}, i_{4}\right\}$ be a subset of $\mathcal{A}$ with $i_{1}<i_{2}<i_{3}<i_{4}$. Let $\mathbf{M}_{J}$ be the submatrix of $\mathbf{M}$ as in Notation 2.4, and denote $D_{J}=\operatorname{det}\left(\mathbf{M}_{J}\right) \in \mathbb{Z}[x]$.

If $f \in \mathbb{K}[x]$ has double roots $a_{1}, a_{2} \in \mathbb{K}^{*}$ with $\operatorname{val}\left(a_{1}\right) \leq \operatorname{val}\left(a_{2}\right)$, then $f\left(a_{1} x\right)$ has double roots $1, b=a_{2} / a_{1}$ and $\operatorname{val}(b) \geq 0$. Hence, we can assume that $f$ has nodes at 1 and $b$, where $b \neq 0,1$ and $\operatorname{val}(b) \geq 0$. Clearly, $f$ has nodes at 1 and $b$ if and only if its vector of coefficients $c$ lies in the kernel of the matrix $\mathbf{M}(b)$.

Expression (1) in Theorem 2.6 explains why it is not enough to consider the combinatorial information given by $w \in \mathcal{T}(\operatorname{ker}(\mathbf{M}(b))$, as the picture is completed by the valuations of the maximal minors of $\mathbf{M}(b)$, which depend on arithmetic conditions on the chosen columns $J$ and algebraic conditions on $b$. Many technical properties of these matrices and, particularly, of its $4 \times 4$ minors are studied in Appendix B.

3.2. Cones of types $I, I I$ and $I I I$. We now give the precise definitions of cones of type I, II and III. The tropical discriminant $\mathcal{T}\left(\Delta_{n}\right)$, that is, the tropicalization of the variety of polynomials $f \in \mathbb{K}[x]$ of degree $n$ with a double root is known to consist of maximal dimensional cones of the secondary fan of the configuration $\mathcal{A}$. Every cone of $\mathcal{T}\left(\Delta_{n}\right)$ corresponds naturally with a marked subdivision of $\mathcal{A}$. The maximal cones of $\mathcal{T}\left(\Delta_{n}\right)$ (which have dimension $n$ ) correspond to the subdivisions of $\mathcal{A}$ that have exactly one marked cell with exactly one marked point. We refer to [5], 9], 6] for basic results on tropical discriminants. On the other hand, we will see that not every $n-1$ dimensional cone given by the intersection of two maximal cones in $\mathcal{T}\left(\Delta_{n}\right)$ belongs to $\mathcal{T}\left(S e v_{n}^{2}\right)$, as there are exceptional configurations (see Proposition 4.6.

Remark 3.2. Let $w \in \mathcal{T}\left(S e v_{n}^{2}\right)$. As $w$ is in the discriminant variety, the subdivision induced by $w$ has at least one marked cell. Moreover, if $w$ is a point in the interior of a maximal cone of $\mathcal{T}\left(S e v_{n}^{2}\right)$, the Newton diagram has at most two marked points because the maximal cones have codimension 2 and every marked point in the Newton diagram imposes a linear restriction that lowers the dimension.

Given a configuration $J=\left\{i_{1}, \ldots, i_{k}\right\}$ and $s \in \mathbb{Z}$, we will use the notation $J(+s)$ for the set $\left\{i_{1}+s, \ldots, i_{k}+s\right\}$ (called a translation) and $J(\times s)$ (when $s \in \mathbb{N}$ ) for the set $\left\{s i_{1}, \ldots, s i_{k}\right\}$.

Definition 3.3. Consider a regular subdivision $\Pi$ with exactly two different marked cells (each with only one marked point). Let $C_{\Pi}$ be the closure of the set of $w \in \mathbb{R}^{n+1}$ with $\Pi_{w}=\Pi$. Then, $C_{\Pi}$ is a cone and we say that it is a cone of type $I$.

Example 3.4. Let $n=5, \mathbb{K}=\mathbb{C}\{\{t\}\}$ with the usual valuation and

$$
f=t(x-1)^{2}(x-t)^{2}\left(x-t^{-1}\right) \text {. }
$$

Them $f \in \mathbb{K}[x]_{w}$, where $w=(2,1,0,0,0,1)$. The Newton diagram of $w$ is displayed on the Left in Figure 1 . Thus, $w$ lies in the interior of the cone of type $I$ induced by the marked subdivision $\{\{0,1,2\},\{2,3,4\},\{4,5\}\}$.

To introduce cones of type $I I$, we need to specify what we understand by an exceptional configuration. 
Definition 3.5. A marked cell $\left\{i_{1}, i_{2}, i_{3}, i_{4}\right\}$ with two marked points is called an exceptional configuration if it is one of the following: $\{0,1,2,3\},\{0,1,2,4\},\{0,2,3,4\}$, $\{0,3,4,6\}$ and $\{0,2,3,6\}$.

We now define type $I I$ cones:

Definition 3.6. Consider a regular subdivision $\Pi$ with exactly one marked cell $\left\{i_{1}, i_{2}, i_{3}, i_{4}\right\}$ with two marked points, which is not the image under a translation of an exceptional configuration. Let $C_{\Pi}$ be the closure of the set of $w \in \mathbb{R}^{n+1}$ with $\Pi_{w}=\Pi$. Then, $C_{\Pi}$ is a cone and we say that it is a cone of type $I I$.

Example 3.7. Let $n=5, \mathbb{K}=\mathbb{C}\{\{t\}\}$ with the usual valuation and

$$
f=(x-1)^{2}(x+2+\sqrt{3}+t)^{2}\left(x-t^{2}\right) .
$$

Then $f \in \mathbb{K}[x]_{w}$, with $w=(2,0,0,1,0,0)$. The Newton Diagram of $w$ is displayed in the Center in Figure 1. Hence, $w$ lies in the interior of the cone of type $I I$ induced by the marked subdivision $\{\{0,1\},\{1,2,4,5\}\}$.

Note that if the double root different from 1 has valuation 0 and independent coefficient other than $-2 \pm \sqrt{3}$ and -1 , the vector of valuations of the coefficients of the polynomial is in a cone of type $I I$, but not in its interior. See Remark 5.2 for an expanded explanation of the choice of $-2 \pm \sqrt{3}$.

Type III cones are not cones of the secondary fan of $\mathcal{A}$ as they also show additional arithmetic constraints:

Definition 3.8. Let $\sigma=\left\{i_{1}, i_{2}, i_{3}\right\}$ and $\tau=\left\{j_{1}, j_{2}\right\}$ with $i_{1}, i_{2}, i_{3}, j_{1}, j_{2}$ different points of $\mathcal{A}$, which satisfy the following restrictions: $g=\operatorname{gcd}\left(i_{3}-i_{1}, i_{2}-i_{1}\right)>1$, and there exists a divisor $d>1$ of $g$ which does not divide $j_{1}-i_{1}$ nor $j_{2}-i_{1}$. Moreover, let $\Pi$ be a regular subdivision of $\mathcal{A}$ with $\sigma$ as the unique marked cell. Let $C=C(\Pi, \tau)$ be the closure of the set of $w \in \mathbb{R}^{n+1}$ which verify:

i) $\Pi_{w}=\Pi$.

ii) Let $\eta$ be the interior normal of the lower facet of $\Gamma_{w}$ corresponding to the marked cell. The minimum value of the scalar products $\left\langle\eta,\left(j, w_{j}\right)\right\rangle$ over all $j$ with $j-i_{1} \not \equiv 0 \bmod d$, is attained at $j_{1}, j_{2}$.

Then, $C$ is a cone and we say it is a cone of type $I I I$ with a hidden tie in $\tau$.

Remark 3.9. Note that it is not enough to ask $d$ to be a prime number in Definition 3.8. Consider for instance the case $\sigma=\{0,4,8\}, \tau=\{2,5\}, g=4$. As we require that $d$ does not divide $2-0$ and $5-0, d=4$ is the only possible value of a divisor $d>1$ of $g$ to get a cone of type III.

Example 3.10. Let $n=5, \mathbb{K}=\mathbb{C}\{\{t\}\}$ with the usual valuation and

$$
f=(x-1)^{2}(x+1+t)^{2}\left(x-t^{2}\right) .
$$

Then, $f \in \mathbb{K}[x]_{w}$, where $w=(2,0,1,0,1,0)$. We have displayed the Newton Diagram of $w$ and the hidden tie on the Right in Figure 1. Thus, $w$ lies in the cone of type $I I I$ induced by the marked subdivision $\Pi=\{\{0,1\},\{1,3,5\}\}$ and the hidden tie in $\tau=\{2,4\}$.

\section{NECESSARY CONDITIONS}

In this section, we will show that $\mathcal{T}\left(S e v_{n}^{2}\right) \subset \mathbb{R}^{n+1}$ is contained in the union of all cones of types $I, I I$ and $I I I$, which proves one of the inclusions in the statement of Theorem 1.1.

Given $w \in \mathrm{Im}_{\mathrm{val}}$, we first state some results that relate the roots of a polynomial $g \in \mathbb{K}[x]_{w}$, and roots of its associated residual polynomials in $K[x]$. For an element $a$, we denote by $\operatorname{mult}(a, g)$ the multiplicity of $a$ as a root of $g$. 
Definition 4.1. Given $w \in \mathbb{R}^{n+1}$, let $\sigma$ be a cell in $\Pi_{w}$. For $g=\sum_{i=0}^{n} d_{i} x^{i} \in \mathbb{K}[x]_{w}$, write $d_{i}=\delta_{i} t^{w_{i}}+*\left(\right.$ so all $\left.\delta_{i} \neq 0\right)$. We say that $g_{\sigma}=\sum_{i \in \sigma} \delta_{i} x^{i} \in K[x]$ is the residual polynomial of $g$ with respect to $\sigma$.

The following proposition is a generalization of the classical Newton-Puiseux theorem on the valuation of the roots of a polynomial when the polynomial has multiple roots. It is a direct consequence of results in [15] and [4].

Proposition 4.2. Let $g=\sum_{i=0}^{n} d_{i} x^{i}$ be a polynomial in $\mathbb{K}[x]$ with $d_{0} \neq 0 \neq d_{n}$ and let $a_{1}, \ldots, a_{n}$ be the roots of $g$ (repeated according to their multiplicities). Let $w \in \mathbb{R}^{n+1}$ be the vector $w=\operatorname{val}(d)$. Using notation from Definition 4.1

i) There exists $\sigma$ a cell of $\Pi_{w}$ such that the lower facet in the Newton Diagram of $w$ that induces $\sigma$ has lattice length $\ell$ and slope $-v$, if and only if the set $J_{\sigma}=\left\{j \in\{1, \ldots, n\}: \operatorname{val}\left(a_{j}\right)=v\right\}$ has cardinal $\ell>0$.

ii) Denote $a_{j}=\alpha_{j} t^{v}+*$ for all $j \in J_{\sigma}$. Then $\left\{\alpha_{j}: j \in J_{\sigma}\right\}$ is the set of nonzero roots of $g_{\sigma}$ and, for all $j \in J_{\sigma}$, $\operatorname{mult}\left(\alpha_{j}, g_{\sigma}\right)=\sum \operatorname{mult}\left(a_{k}, g\right)$ where the sum is over all $k \in J_{\sigma}$ such that $\alpha_{k}=\alpha_{j}$.

iii) For all $j \in J_{\sigma}$, both the multiplicity of $a_{j}$ as a root of $g$ and the multiplicity of $\alpha_{j}$ as a root of $g_{\sigma}$ are strictly smaller than the cardinal of the cell $\sigma$.

Proof. Items i) and ii) were proved in Proposition 1.8 and Proposition 1.9 on [15] respectively.

To see item iii), Theorem 5.6 in [4] states in the case of univariate polynomials that, if we fix $\kappa \in \mathbb{N}, g$ has a root of multiplicity at least $\kappa+1$ if and only if there exists $w \in \mathbb{R}^{n+1}$ with the following property: for any $J \subset \mathcal{A}$ with $\# J \leq \kappa$, the minimum of the scalar products $\langle w, \cdot\rangle$ is attained at least twice on $\mathcal{A} \backslash J$. Using this result and item ii), if there is a root $a_{j}$ of $g$ of multiplicity $\tau, \alpha_{j}$ is a root of $g_{\sigma}$ of multiplicity at least $\tau$, and therefore removing any subset $J \subset \sigma$ of $\tau$ elements, $\sum_{i \in \sigma-J} \delta_{i} x^{i}$ cannot be a monomial. It follows that the the cardinal of $\sigma$ has to be at least $\tau+1$.

As a straightforward consequence of Proposition 4.2, we see that:

Corollary 4.3. Let $w \in \mathbb{R}^{n+1}$ be a point such that the subdivision $\Pi_{w}$ has only one marked cell of lattice length 3. Then, $w$ does not lie in $\mathcal{T}\left(S e v_{n}^{2}\right)$.

Another consequence of Proposition 4.2 is the following:

Corollary 4.4. Let $f \in \mathbb{K}[x]_{w}$, with $w$ an element in the interior of a cone of type $I, I I$ or III. Let $a=\alpha t^{\operatorname{val}(a)}+*, b=\beta t^{\mathrm{val}(b)}+*$ be two different multiple roots of f. If $\operatorname{val}(a)=\operatorname{val}(b)$, then $\alpha \neq \beta$.

Proof. Since $\operatorname{val}(a)=\operatorname{val}(b)$, by item $i i)$ of Proposition 4.2 both $\alpha$ and $\beta$ are multiple roots of the residual polynomial associated to the marked cell of slope $-\operatorname{val}(a)$ in the Newton diagram of $w$. Moreover, if $\alpha=\beta$, then by the same item it is root of multiplicity at least four. By item iii), this means that the corresponding marked cell needs to have at least five points (or equivalently, three marked points). But then, $w$ cannot be in the interior of a cone of type $I, I I, I I I$, which is a contradiction. Then, $\alpha \neq \beta$.

Remark 4.5. Given $f \in \mathbb{K}[x]$, we have already remarked that we assume that one of its roots is equal to 1 . Moreover, by item $i$ ) in Proposition 4.2, this means we can assume a desired lower facet from the Newton Diagram to have slope 0. Multiplying $f$ by an appropriate constant in $\mathbb{K}^{*}$, we can moreover assume without loss of generality that $\operatorname{val}\left(c_{i}\right) \geq 0$ for all coefficients of $f$ and $\operatorname{val}\left(c_{j}\right)=0$ for every $j \in \mathcal{A}$ that lie in the cell associated to the lower facet of slope 0 . 
In the definition of type $I I$ cones, we explicitly excluded subdivisions whose marked cell is a translation of an exceptional configuration. We now show that these subdivisions with exceptional configurations are not in $\mathcal{T}\left(S e v_{n}^{2}\right)$.

Proposition 4.6. Let $\sigma=\left\{i_{1}, i_{2}, i_{3}, i_{4}\right\}$ be the translation of an exceptional configuration. Let $w \in \operatorname{Im}_{\mathrm{val}}$ be a point such that $\sigma$ is the only marked cell from the subdivision of $\mathcal{A}$ induced by $w$. Then $w$ is not in $\mathcal{T}\left(S e v_{n}^{2}\right)$.

Proof. As the lattice length of the marked cell has to be at least 4, any $w$ such that the induced subdivision has as the only marked cell a translation of $\{0,1,2,3\}$ is not in $\mathcal{T}\left(S e v_{n}^{2}\right)$.

As we will consider the nonzero roots of residual polynomials with support in $\sigma$, without loss of generality we can assume $\sigma$ an exceptional configuration. Also, we can assume that the lower facet associated to $\sigma$ has slope 0. Suppose there is a polynomial $f \in \mathbb{K}[x]_{w} \cap S e v_{n}^{2}$ and $1, b$ are its double roots. As $\sigma$ is the only marked cell, $\operatorname{val}(b)=0$ and $b=\beta+*$ where, as in the proof of Corollary $4.4, \beta \neq 1$.

If $\sigma=\{0,1,2,4\}$ and $f_{\sigma}(x)=\gamma_{4}(x-1)^{2}(x-\beta)^{2}=\sum_{i \in \sigma} \gamma_{i} x^{i}$, then $\gamma_{3}=\frac{\gamma_{1}}{\beta}$ and moreover, $\gamma_{3}=0$ and $\gamma_{1} \neq 0$, which is a contradiction.

If $\sigma=\{0,2,3,6\}$ and $f_{\sigma}(x)=\gamma_{6}(x-1)^{2}(x-\beta)^{2}\left(x^{2}+\delta x+\epsilon\right)=\sum_{i \in \sigma} \gamma_{i} x^{i}$, as $\beta \gamma_{2} \gamma_{3}$ is a linear combination with coefficients in $\mathbb{Q}[\beta, \delta, \epsilon]$ of the coefficients of the monomials $x, x^{4}, x^{5}$ (see Computation C.1), $\beta \gamma_{2} \gamma_{3}=0$ which is also a contradiction.

Having ruled out these two configurations and considering the polynomial $g \in$ $\mathbb{K}[x] \cap S e v_{n}^{2}$ define as $g(x)=x^{n} f\left(x^{-1}\right)$, we discard the remaining exceptional configurations because $\{0,2,3,4\}=\{4-i: i \in\{0,1,2,4\}\}$ and $\{0,3,4,6\}=\{6-i$ : $i \in\{0,2,3,6\}\}$.

We now provide another technical result related to cones of type $I I I$.

Proposition 4.7. Let $n \geq 4$ and $w \in \mathcal{T}\left(S e v_{n}^{2}\right)$ such that the subdivision induced by $w$ has only one marked cell $\sigma=\left\{i_{1}, i_{2}, i_{3}\right\}$. Let $f \in S e v_{n}^{2} \cap \mathbb{K}[x]_{w}$ and $a=$ $\alpha t^{v}+*, b=\beta t^{v}+* \in \mathbb{K}^{*}$ be multiple roots of $f$. Then $\frac{\beta}{\alpha} \neq 1$ is a root of unity of order that divides $\operatorname{gcd}\left(i_{2}-i_{1}, i_{3}-i_{1}\right)$.

Proof. We can assume as before that $a=1$, and $i_{1}=0$. Let $f_{\sigma}=\gamma_{0}+\gamma_{i_{2}} x^{i_{2}}+$ $\gamma_{i_{3}} x^{i_{3}}$ be the residual polynomial of $f$ associated to the marked cell $\sigma$. Then by Proposition 4.2 1, $\beta$ are double roots of $f_{\sigma}$ and $\beta \neq 1$. By evaluating we obtain the following equalities:

$$
\begin{array}{cccc}
\text { i) } & \gamma_{0}+\gamma_{i_{2}}+\gamma_{i_{3}}=0 . & \text { iii }) & \gamma_{0}+\gamma_{i_{2}} \beta^{i_{2}}+\gamma_{i_{3}} \beta^{i_{3}}=0 . \\
\text { ii) } & i_{2} \gamma_{i_{2}}+i_{3} \gamma_{i_{3}}=0 . & \text { iv }) & i_{2} \gamma_{i_{2}} \beta^{i_{2}}+i_{3} \gamma_{i_{3}} \beta^{i_{3}}=0 .
\end{array}
$$

By equalities $i$ ) and $i i)$ we have $\gamma_{0}=\left(\frac{i_{3}}{i_{2}}-1\right) \gamma_{i_{3}}$ and $\gamma_{i_{2}}=-\frac{i_{3}}{i_{2}} \gamma_{i_{3}}$. Replacing in equality $i v$ ) and simplifying we obtain $\beta^{i_{2}}=\beta^{i_{3}}$. Finally, using equality iii) we obtain $\beta^{i_{3}}=1$. It follows that $\beta$ is an $d$-th primitive root of unity where $d \neq 1$ and $d$ divides $\operatorname{gcd}\left(i_{2}, i_{3}\right)$.

Finally we can prove that, for $w \in \mathbb{R}^{n+1}$ to be a point in $\mathcal{T}\left(S e v_{n}^{2}\right)$, is a necessary condition that $w$ be a point in the union of all cones of type $I, I I$ or $I I I$. We start by defining an open dense subset $\mathcal{V}$ of $\operatorname{Sev}_{n}^{2}(\mathbb{K})$. Consider the open dense subset $\mathcal{U}^{\prime}$ of $\mathcal{U}$ given by those $\left(a, b, a_{1}, \ldots, a_{n-3}\right)$ such that $D_{J}(a / b) \neq 0$, for any $J \subset \mathcal{A}$ of cardinal 4 . Note that $D_{J}(a / b) \neq 0$ if and only if $D_{J}(b / a) \neq 0$ by Lemma B.1. Moreover, $\mathcal{U}^{\prime} \neq \emptyset$ by Lemma B.2. We call $\mathcal{V}=\varphi_{\mathbb{K}}\left(\mathcal{U}^{\prime}\right)$, where the map $\varphi_{K}$ has been defined in Section 3.1 .

Theorem 4.8. Let $n \geq 4$ and $w \in \mathbb{R}^{n+1}$ be in a maximal cone of $\mathcal{T}\left(\operatorname{Sev}_{n}^{2}\right)$. Then $w$ is in a cone of type $I$, II or III. 
Proof. Without loss of generality, we assume that $w \in \operatorname{Im}_{\text {val }}$ and that there are one or two marked points in the subdivision induced by the Newton Diagram of $w$. If there are two marked points in different cells of the subdivision, then $w$ is in a cone of type $I$. In the same way, if there are two marked points in only one marked cell of the subdivision, by Proposition 4.6, we know the marked cell is not the translation of an exceptional configuration, hence $w$ is in a cone of type $I I$.

Let us consider now the remaining case of only one marked cell $\sigma=\left\{i_{1}, i_{2}, i_{3}\right\}$ with one marked point. There is a polynomial $f \in S e v_{n}^{2} \cap \mathbb{K}[x]_{w}$ with $a, b \in \mathbb{K}^{*}$ double roots. Then, because of item iii) in Proposition 4.2 val $(a)=\operatorname{val}(b)$. To see that $w$ is in a cone of type $I I I$, we assume without loss of generality that $a=1$ and $b=\beta+h t^{v}+*$, with $v>0$. Using Proposition 4.7 we know that $\beta$ is a root of unity of order $d$, where $d>1$ divides $\operatorname{gcd}\left(i_{2}-i_{1}, i_{3}-i_{1}\right)$. Also, we assume without loss of generality that $w \in \operatorname{val}(\mathcal{V})$. For any index $k \notin \sigma$, we have by Lemma B.4 that $D_{\sigma \cup\{k\}}(\beta)=0$. Since we assume that $D_{\sigma \cup\{k\}}(b) \neq 0$, it follows that $h \neq 0$.

As $a=1$, we can assume $w_{i_{1}}=w_{i_{2}}=w_{i_{3}}=0$. We want to prove that $\min _{j \in J}\left\{w_{j}\right\}$ is attained at least twice, where $J=\left\{j \in \mathcal{A}: d \nmid j-i_{1}\right\}$. Suppose that the minimum is only attained once at $i_{4} \in J$, (with $w_{i_{4}}>0$ ), and let $S=\{j \in$ $\left.\mathcal{A}: d \mid j-i_{1}\right\}$. Because of Lemma B.5 $\operatorname{val}\left(D_{\sigma \cup\{j\}}(b)\right)=v$ for all $j \in J$, hence $\min _{j \in J}\left\{\operatorname{val}\left(D_{\sigma \cup\{j\}}(b)\right)+w_{j}\right\}$ is also only attained once at $i_{4}$. Then, by Theorem 2.6 there exists at least one $s_{0} \in S$ such that, for all $k \in \mathcal{A}, \operatorname{val}\left(D_{\sigma \cup\left\{s_{0}\right\}}(b)\right)+w_{s_{0}} \leq$ $\operatorname{val}\left(D_{\sigma \cup\{k\}}(b)\right)+w_{k}$.

Consider the set $I=\left\{i_{1}, i_{2}, s_{0}\right\}$. Again by Theorem 2.6 the minimum in the set $\left\{\operatorname{val}\left(D_{I \cup\{k\}}(b)\right)+w_{k}: k \in \mathcal{A}\right\}$ is attained at least twice. However, again by Lemma B.5. $\operatorname{val}\left(D_{I \cup\{s\}}(b)\right)=4 v=\operatorname{val}\left(D_{\sigma \cup\left\{s_{0}\right\}}(b)\right)$ when $s \in S \backslash I$ and $\operatorname{val}\left(D_{I \cup\{j\}}(b)\right)=v=\operatorname{val}\left(D_{\sigma \cup\{j\}}(b)\right)$ when $j \in J$. Then

$\operatorname{val}\left(D_{I \cup\{k\}}(b)\right)+w_{k}=\left\{\begin{array}{lr}\operatorname{val}\left(D_{\sigma \cup\left\{s_{0}\right\}}(b)\right)+w_{i_{3}}=\operatorname{val}\left(D_{\sigma \cup\left\{s_{0}\right\}}(b)\right)=: D_{i_{3}} & \text { if } k=i_{3} \\ \operatorname{val}\left(D_{\sigma \cup\left\{s_{0}\right\}}(b)\right)+w_{k}>D_{i_{3}} & \text { if } k \in S \backslash\left(I \cup\left\{i_{3}\right\}\right) \\ \operatorname{val}\left(D_{\sigma \cup\{k\}}(b)\right)+w_{k} \geq \operatorname{val}\left(D_{\sigma \cup\left\{s_{0}\right\}}(b)\right)+w_{s_{0}}>D_{i_{3}} & \text { if } k \in J \\ \infty>D_{i_{3}} & \text { if } k \in I\end{array}\right.$

so the minimum would only be attained at $k=i_{3}$, which is a contradiction.

\section{SufFicient CONDitions}

In this section we prove that each of the cones of type $I, I I$ and $I I I$ belongs to the tropical Severi variety. Our proof is constructive, that is, it is done showing that, for every $w$ in one of the cones, we can compute a polynomial $f \in \mathbb{K}[x]_{w} \cap S e v_{n}^{2}$.

The key is to choose an appropriate configuration $J=\left\{i_{1}, i_{2}, i_{3}, i_{4}\right\}$ and study the matrix $\mathbf{M}_{J}(x)$ as well as the valuation of its determinant $D_{J}=\operatorname{det}\left(\mathbf{M}_{J}\right)$, where the matrix $\mathbf{M}$ is as in Definition 3.1. We will need many technical lemmas, that are collected in Appendix $B$. In particular, we have that $D_{J} \in \mathbb{Z}[x]-\{0\}$ for all 4-index set $J$, by Lemma B.2.

We want to prove that there exists $c=\left(c_{0}, \ldots, c_{n}\right)$ with val $(c)=w$ and $b$ different from 1 that solve the system $\mathbf{M}(b) c^{T}=0$, and therefore $w$ is a point on $\mathcal{T}\left(S e v_{n}^{2}\right)$. Moreover, we are going to find $f \in \mathbb{K}[x]_{w}$ with multiple roots 1 and $b$ such that $c_{i}=t^{w_{i}}$ for all $i \notin J$. Then, we are looking for an appropriate $b$ and $\left(c_{i_{1}}, c_{i_{2}}, c_{i_{3}}, c_{i_{4}}\right)$ with each of its coordinates of the correct valuation that solve the equation system:

$$
\mathbf{M}_{J}(b) \cdot\left(c_{i_{1}}, c_{i_{2}}, c_{i_{3}}, c_{i_{4}}\right)^{T}=-\left(\sum_{i \notin J} t^{w_{i}}, \sum_{i \notin J} i t^{w_{i}}, \sum_{i \notin J} b^{i} t^{w_{i}}, \sum_{i \notin J} i b^{i} t^{w_{i}}\right)^{T}
$$

The easiest case are cones of type $I$. 
5.1. Type $I$. Consider $w \in \operatorname{Im}_{\text {val }}$ a point in the interior of a cone of type $I$. That is, the subdivision induced by $w$ has two different marked cells with one marked point each. Without loss of generality, we can assume that the slopes of the lower facets from the Newton diagram corresponding to those marked cells are 0 and $-v$ where $v>0$. Then, the Newton diagram is as in Figure 5 .

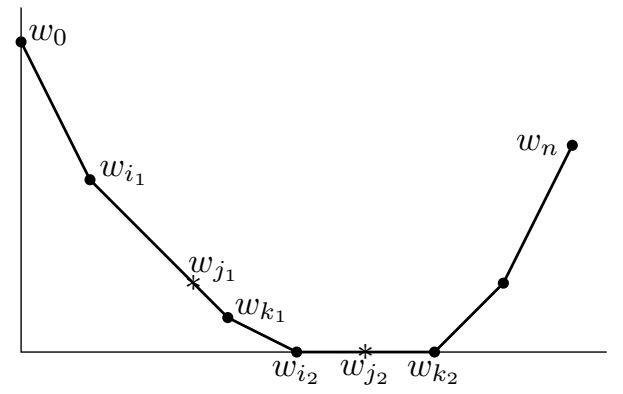

FiguRE 5. Two marked cells with different slopes

That is, there are two marked cells, $\left\{i_{1}, j_{1}, k_{1}\right\}$ and $\left\{i_{2}, j_{2}, k_{2}\right\}$ where $k_{1} \leq i_{2}$, in the corresponding subdivision. We can assume $w_{i_{2}}=w_{j_{2}}=w_{k_{2}}=0$. Let $-v=\frac{w_{k_{1}}-w_{i_{1}}}{k_{1}-i_{1}}$ be the slope of the non-horizontal marked segment.

Theorem 5.1. Let $n \geq 4$ and $w \in \operatorname{Im}_{\mathrm{val}}$ be a point in a cone of type $I$. Then $w$ is an element of $\mathcal{T}\left(S e v_{n}^{2}\right)$.

Proof. Using the same notation and assumptions as before, we are going to present a polynomial $f$ complying with the request. In particular, we can assume $w$ is a point in the interior of the cone. It is worth mentioning that this is only one choice but there are infinite polynomials that satisfy the theorem (see [13]). Moreover, if $w \in \mathbb{Z}^{n+1}$, then our proposed solution $f$ lies in $\mathbb{C}(t)[x]$.

We will build a polynomial $f=\sum_{i=0}^{n} c_{i} x^{i} \in S e v_{n}^{2}$ such that $f \in \mathbb{K}[x]_{w}$ and its double roots are $a=1$ and $b=t^{v}$. Let $J=\left\{i_{1}, j_{1}, j_{2}, k_{2}\right\}$ and take $c_{l}=t^{w_{l}}$ for all index $l \notin J$.

We want to see that there are $c_{j_{2}}, c_{k_{2}}$ of valuation 0 and $c_{i_{1}}, c_{j_{1}}$ of valuations $w_{i_{1}}$ and $w_{j_{1}}$ respectively that are solutions of the system in Equation (4). Let us see that there exist $\gamma_{i_{1}}, \gamma_{i_{2}}$ such that $c_{i_{1}}=\gamma_{i_{1}} t^{w_{i_{1}}}+*, c_{j_{1}}=\gamma_{j_{1}} t_{j_{1}}+*$ and $\gamma_{j_{2}}, \gamma_{k_{2}}$ with $c_{j_{2}}=\gamma_{j_{2}}+*$ and $c_{k_{2}}=\gamma_{j_{1}}+*$. Replacing this notation in Equation (4), we get the system

$$
\left(\begin{array}{cccc}
t^{w_{i_{1}}} & t^{w_{j_{1}}} & 1 & 1 \\
i_{1} t^{w_{i_{1}}} & j_{1} t^{w_{j_{1}}} & j_{2} & k_{2} \\
t^{w_{i_{1}}+v i_{1}} & t^{w_{j_{1}}+v j_{1}} & t^{v j_{2}} & t^{v k_{2}} \\
i_{1} t^{w_{i_{1}}+v i_{1}} & j_{1} t^{w_{j_{1}}+v j_{1}} & j_{2} t^{v j_{2}} & k_{2} t^{v k_{2}}
\end{array}\right)\left(\begin{array}{c}
\gamma_{i_{1}} \\
\gamma_{j_{1}} \\
\gamma_{j_{2}} \\
\gamma_{k_{2}}
\end{array}\right)=\left(\begin{array}{c}
-1+* \\
-i_{2}+* \\
-t^{w_{k_{1}}+v k_{1}}+* \\
-k_{1} t^{w_{k_{1}}+v k_{1}}+*
\end{array}\right)
$$

Dividing the third and fourth rows of the $4 \times 4$ matrix by $t^{v k_{1}+w_{k_{1}}}$ we obtain a system where every term has nonnegative valuation because $i_{1}, j_{1}, k_{1}$ are the indices where the minimum is attained in the segment with slope $-v$. Moreover, the valuation of the entries in those rows associated to $j_{2}$ and $k_{2}$ is positive because of the convexity of the Newton Diagram.

Setting $t=0$ we obtain the $4 \times 4$ invertible system over $K$ (in matrix form):

$$
\left(\begin{array}{cccc}
0 & 0 & 1 & 1 \\
0 & 0 & j_{2} & k_{2} \\
1 & 1 & 0 & 0 \\
i_{1} & j_{1} & 0 & 0
\end{array}\right)\left(\begin{array}{c}
\gamma_{i_{1}} \\
\gamma_{j_{1}} \\
\gamma_{j_{2}} \\
\gamma_{k_{2}}
\end{array}\right)=\left(\begin{array}{c}
-1 \\
-i_{2} \\
-1 \\
-k_{1}
\end{array}\right)
$$


Hence, system (4) has also a unique solution, which equals:

$\left(c_{i_{1}}, c_{j_{1}}, c_{j_{2}}, c_{k_{2}}\right)=\left(-\frac{j_{1}-k_{1}}{j_{1}-i_{1}} t^{w_{i_{1}}}+*,-\frac{k_{1}-i_{1}}{j_{1}-i_{1}} t^{w_{j_{1}}}+*,-\frac{k_{2}-i_{2}}{k_{2}-j_{2}}+*,-\frac{i_{2}-j_{2}}{k_{2}-j_{2}}+*\right)$,

and has the correct valuations.

5.2. Type $I I$. Type $I I$ cones are the most difficult case to prove completely. We follow the same approach as for type $I$ cones, but we have to separate the case where the marked cell is an affine image of an exceptional configuration. The choice of the node $b$ is more complicated and subtle than in the case of nodes of type $I$.

5.2.1. The general case. For points $w$ in cones of type $I I$ and $I I I$ we will assume that the marked cell has slope 0 and we will build a polynomial $f \in \mathbb{K}[x]_{w}$ with double roots 1 and $b$ of valuation 0 . We need that $b=\beta+*$ and $D_{J}(b) \neq 0$, but this is possible since $D_{J} \neq 0$ (see Lemma B.2). Note that, as $\operatorname{val}(b)=0, \beta \neq 0$. Also, by Corollary 4.4 we can assume $\beta \neq 1$. Multiplying by the adjoint matrix $\operatorname{adj}\left(\mathbf{M}_{J}(b)\right)$ of $\mathbf{M}_{J}(b)$, the solutions for all $1 \leq j \leq 4$ of Equation (4) are

$$
c_{i_{j}}=-\sum_{i \notin J} \pm \frac{D_{(J \cup\{i\})-\left\{i_{j}\right\}}(b)}{D_{J}(b)} t^{w_{i}},
$$

where the signs depend on the number of permutations necessary to reorder from lowest to highest the ordered set $J$ as we replace $i_{j}$ by $i$ (see Notation 2.4). As $\operatorname{val}(b)=0$, the entries of the matrix $\operatorname{adj}\left(\mathbf{M}_{I}(b)\right)$ are of valuation at least 0 . Also, if $\operatorname{val}\left(w_{i}\right)>0$ for every $i \notin J$, then every coordinate in the right hand part of the system has positive valuation. Therefore, if the solution $\left(c_{i_{1}}, c_{i_{2}}, c_{i_{3}}, c_{i_{4}}\right)$ fulfills $\operatorname{val}\left(c_{i_{j}}\right)=0$ for some $1 \leq j \leq 4$, the valuation of $D_{J}(b)$ has to be positive. Hence, $D_{J}(\beta)=0$.

Consider now a point $w$ in the interior of a cone of type $I I$. Then there is one marked cell $J=\left\{i_{1}, i_{2}, i_{3}, i_{4}\right\}$ with two marked points. We can assume $w_{i_{1}}=w_{i_{2}}=$ $w_{i_{3}}=w_{i_{4}}=0$, and $w_{j}>0$ for all $j \notin J$, so we are in the situation stated above.

Remark 5.2. Let $f=(x-1)^{2}(x-b)^{2}(x-a) \in \mathbb{K}[x]$ where $b=\beta+*$ with $\beta \in K \backslash\{0,1\}$ and $\operatorname{val}(a) \neq 0$. From item i) in Proposition 4.2, the subdivision induced by the vector of valuations of the coefficients of $f$ has two cells, one of lattice length 1 and another one of lattice length 4 . Then, for $w$ to be a point in the interior of a cone of type $I I$, the cell of lattice length 4 has to be either $\{0,1,3,4\}$ or $\{1,2,4,5\}$ by Proposition 4.6. It is easy to see that the only zeros in $K \backslash\{0,1\}$ of either $D_{\{0,1,3,4\}}$ or $D_{\{1,2,4,5\}}$ are $-2 \pm \sqrt{3}$, as used in Example 3.7.

In what follows, the expression that a value is "repeated at most once" means that it can occur 1 or 2 times (but not more).

Theorem 5.3. Let $w \in \mathbb{R}^{n+1}$ be a point a cone of type II with marked cell $J=$ $\left\{i_{1}, i_{2}, i_{3}, i_{4}\right\}$. If there is a $\beta \in K \backslash\{0,1\}$ root of $D_{J}$ such that each of the powers $\left\{\beta^{i_{1}}, \beta^{i_{2}}, \beta^{i_{3}}, \beta^{i_{4}}\right\}$ is repeated at most once, then $w$ is in $\mathcal{T}\left(\operatorname{Sev}_{n}^{2}\right)$.

Proof. Without loss of generality, we can assume $w \in \operatorname{Im}_{\mathrm{val}}$ is a generic point in the interior of the cone, and $w_{i_{j}}=0$ for all $1 \leq j \leq 4$. By Lemma B.9 the set $S=\left\{i \in \mathcal{A}-J: D_{(J \cup\{i\})-\left\{i_{1}\right\}}(\beta) \neq 0\right\} \neq \emptyset$. As $w$ is a generic point, we can assume that there exists $i_{5} \in S$ such that $0<w_{i_{5}}<w_{i}$ for all $i \in S-\left\{i_{5}\right\}$. Since $D_{J}(\beta)=0$, by Lemma B.6, $D_{\left(J \cup\left\{i_{5}\right\}\right)-\left\{i_{j}\right\}}(\beta) \neq 0$ for all $1 \leq j \leq 4$. Note that, since $\left\{\beta^{i_{j}}\right\}_{j=1}^{4}$ are not all equal, by Lemma B.7 the multiplicity of $\beta$ as a root of $D_{J}$ is at most two. Then, by Lemma B.8 the multiplicity $m$ of $\beta$ as a root of $D_{J}$ is lower or equal to the multiplicity $m_{e j}$ of $\beta$ as a root of $D_{(J \cup\{e\})-\left\{i_{j}\right\}}$ for all $e \notin S \cup J$ and $1 \leq j \leq 4$. Let $b=\beta+h t^{\frac{w_{i_{5}}}{m}} \in \mathbb{K}$ such that $D_{J}(b) \neq 0$ and $f=\sum_{i=0}^{n} c_{i} x^{i}$ such 
that $c_{i}=t^{w_{i}}$ for all $i \notin J$. We are going to see that the coefficients $\left\{c_{i_{j}}\right\}_{j=1}^{4}$ such that $1, b$ are multiple roots of $f$ fulfill val $\left(c_{i_{j}}\right)=0$ for all $1 \leq j \leq 4$ and therefore $w$ is in $\mathcal{T}\left(S e v_{n}^{2}\right)$. Note that $\operatorname{val}\left(D_{J}(b)\right)=w_{i_{5}}$ and, for all $e \notin S \cup J$ and $1 \leq j \leq 4$, $\operatorname{val}\left(D_{(J \cup\{e\})-\left\{i_{j}\right\}}(b)\right)=m_{e j} \frac{w_{i_{5}}}{m} \geq w_{i_{5}}$. We saw in Equation 5 that the solutions $\left(c_{i_{1}}, c_{i_{2}}, c_{i_{3}}, c_{i_{4}}\right)$ are of the form $c_{i_{j}}=-\sum_{i \notin J} \pm \frac{D_{(J \cup\{i\})-\left\{i_{j}\right\}}(b)}{D_{J}(b)} t^{w_{i}}$ for all $1 \leq j \leq 4$. If we analyze the valuation for every term of that sum, we can see that:

- If $i=i_{5}$, then val $\left(\frac{D_{\left(J \cup\left\{i_{5}\right\}\right)-\left\{i_{j}\right\}}(b)}{D_{J}(b)} c_{i_{5}}\right)=0-w_{i_{5}}+w_{i_{5}}=0$.

- If $i \notin S \cup J$, then val $\left(\frac{D_{(J \cup\{i\})-\left\{i_{j}\right\}}(b)}{D_{J}(b)} c_{i}\right) \geq w_{i_{5}}-w_{i_{5}}+w_{i}>0$ because $w_{i}>0$.

- If $i \in S$, then val $\left(\frac{D_{(J \cup\{i\})-\left\{i_{j}\right\}}(b)}{D_{J}(b)} c_{i}\right)=0-w_{i_{5}}+w_{i}>0$ because $w_{i}>w_{i_{5}}$. Then,

$$
c_{i_{j}}= \pm \frac{D_{\left(J \cup\left\{i_{5}\right\}\right)-\left\{i_{j}\right\}}(b)}{D_{J}(b)} t^{w_{i_{5}}}+*
$$

and has valuation 0 for all $1 \leq j \leq 4$.

5.2.2. Exceptional Configurations. In Theorem B.11 we proved that the sets $\sigma=$ $\left\{i_{1}, i_{2}, i_{3}, i_{4}\right\}$ such that every root $\beta \in K \backslash\{0,1\}$ of $D_{\sigma}(x)$ satisfies that there are at least three of the powers $\left\{\beta^{i_{1}}, \beta^{i_{2}}, \beta^{i_{3}}, \beta^{i_{4}}\right\}$ equal, are precisely those of the form $\sigma=(J(\times s))(+r)=\left\{s j_{1}+r, s j_{2}+r, s j_{3}+r, s j_{4}+r\right\}$ where $s \in \mathbb{N}, r \in \mathbb{Z}_{\geq 0}$ and $J=\left\{j_{1}, j_{2}, j_{3}, j_{4}\right\}$ is an exceptional configuration (see Definition 3.5).

In Proposition 4.6 we already proved that if $w$ is a point such that the subdivision induced by $w$ has only one marked cell $J$ and this cell is a translation of an exceptional configuration, then $w$ is not in $\mathcal{T}\left(S e v_{n}^{2}\right)$. In the next theorem we will see that if the marked cell $\sigma$ is a image under an affine map $(J(\times s))(+r)$ of an exceptional configuration such that $s=\operatorname{gcd}\left(i_{4}-i_{1}, i_{3}-i_{1}, i_{2}-i_{1}\right)>1$, then $w$ is in fact in $\mathcal{T}\left(S e v_{n}^{2}\right)$. With this result we complete the proof that all $w$ in the interior of a type $I I$ cone is in $\mathcal{T}\left(S e v_{n}^{2}\right)$.

Theorem 5.4. Let $J=\left\{j_{1}, j_{2}, j_{3}, j_{4}\right\}$ be an exceptional configuration. Let $\Pi$ be a subdivision of $\mathcal{A}$ such that the only marked cell is $I=\left\{i_{1}, i_{2}, i_{3}, i_{4}\right\}$ where $I=$ $(J(\times s))(+r)$ for $s \in \mathbb{N}$ and $r \in \mathbb{Z}_{\geq 0}$. Let $w \in \operatorname{Im}_{\mathrm{val}} \subseteq \mathbb{R}^{n+1}$ be a point in the cone $C_{\Pi}$ defined by the closure of the set of points in $\mathbb{R}^{n+1}$ whose Newton diagram induces the subdivision $\Pi$. If $s>1$, then $w$ is a point in $\mathcal{T}\left(\operatorname{Sev}_{n}^{2}\right)$.

Proof. We can assume that $w \in \operatorname{Im}_{\mathrm{val}}$ is a generic point in the interior of the cone and that $w_{i_{1}}=w_{i_{2}}=w_{i_{3}}=w_{i_{4}}=0$. We may also assume without loss of generality that $i_{5}$ is the only index where $\min \left\{w_{i}: i \notin I\right.$ and $\left.s \nmid i\right\}$ is attained. Let $\beta \in K \backslash\{0,1\}$ be a primitive $s$-th root of unity and $f=\sum_{i=0}^{n} c_{i} x^{i} \in \mathbb{K}[x]$ such that $c_{i}=t^{w_{i}}$ for all $i \notin I$. We want to find $\left\{c_{i}\right\}_{i \in I} \subseteq \mathbb{K}$ such that $\operatorname{val}\left(c_{i}\right)=0$ for all $i \in I$ and $f$ has two multiple roots 1 and $b=\beta+*$. Take $b=\beta+h t^{v}$, such that $h \in K^{*}, v=\frac{w_{i_{5}}}{3}$ and $D_{I}(b) \neq 0$. Then the system in Equation 4 has a unique solution in $\mathbb{K}^{4}$ (presented in Equation (5)).

By Lemma B.5 we know that $\operatorname{val}\left(D_{I}(b)\right)=4 v$, and that for every $i \notin I$ $\operatorname{val}\left(D_{I \cup\{i\}-\left\{i_{j}\right\}}(b)\right)=v$ if $s \mid i$ and $\operatorname{val}\left(D_{I \cup\{i\}-\left\{i_{j}\right\}}(b)\right)=4 v$ if $s \nmid i$. Then,

- $\operatorname{val}\left(\frac{D_{(I \cup\{i\})-\left\{i_{j}\right\}}(b)}{D_{I}(b)} t^{w_{i}}\right)=v-4 v+w_{i}=w_{i}-3 v$ if $s \nmid i$.

- $\operatorname{val}\left(\frac{D_{(I \cup\{i\})-\left\{i_{j}\right\}}(b)}{D_{I}(b)} t^{w_{i}}\right)=4 v-4 v+w_{i}=w_{i}$ if $s \mid i$.

Since $v=\frac{w_{i_{5}}}{3}$, we have that: 
- $\operatorname{val}\left(\frac{D_{\left(I \cup\left\{i_{5}\right\}\right)-\left\{i_{j}\right\}}(b)}{D_{I}(b)} t^{w_{i_{5}}}\right)=w_{i_{5}}-3 v=w_{i_{5}}-3 \frac{w_{i_{5}}}{3}=0$.

- $\operatorname{val}\left(\frac{D_{(I \cup\{i\})-\left\{i_{j}\right\}}(b)}{D_{I}(b)} t^{w_{i}}\right)=w_{i}-3 v=w_{i}-w_{i_{5}}>0$ if $s \nmid i$ because of the choice of $i_{5}$.

- $\operatorname{val}\left(\frac{D_{(I \cup\{i\})-\left\{i_{j}\right\}}(b)}{D_{I}(b)} t^{w_{i}}\right)=w_{i}>0$ if $s \mid i$.

Therefore, we have that $\min _{i \notin I}\left\{\operatorname{val}\left(\frac{D_{(I \cup\{i\})-\left\{i_{j}\right\}}(b)}{D_{I}(b)} t^{w_{i}}\right)\right\}$ is attained only at $i_{5}$ where is zero, hence $\operatorname{val}\left(c_{i_{j}}\right)=0$ for all $1 \leq j \leq 4$.

5.3. Type $I I I$. Finally, consider $w \in \mathbb{R}^{n+1}$ a point in the interior of a cone of type $I I I$. That is, $w$ is such that the induced subdivision has only one marked cell $\left\{i_{1}, i_{2}, i_{3}\right\}$ with one marked point $i_{2}$, and a hidden tie $\left\{i_{4}, i_{5}\right\}$. Assume also, without loss of generality, that $w_{i_{j}}=0$ for all $1 \leq j \leq 3$ and $w_{k}>0$ for all $k \notin I$. Then:

- $\operatorname{gcd}\left(i_{2}-i_{1}, i_{3}-i_{1}\right)>1$.

- There is an integer $d>1$, which divides of $\operatorname{gcd}\left(i_{2}-i_{1}, i_{3}-i_{1}\right)$, such that the set $\left\{w_{j}: j \in \mathcal{A}\right.$ and $\left.j \not \equiv i_{1} \bmod d\right\}$ attains its minimum twice at $\left\{i_{4}, i_{5}\right\}$.

Theorem 5.5. Let $w \in \mathbb{R}^{n+1}$ be a point in a cone of type III. Then $w$ is an element of $\mathcal{T}\left(S e v_{n}^{2}\right)$.

Proof. Without loss of generality, we assume $w \in \operatorname{Im}_{\mathrm{val}}$ a generic point in the interior of the cone and $w_{i_{1}}=w_{i_{2}}=w_{i_{3}}=0$ where $\left\{i_{1}, \ldots, i_{5}, d\right\}$ are as in the notation above. Let $\beta$ be a primitive root of unity of order $d$ and $b=\beta+t^{w_{i_{4}}}$. Note that we can write $b^{d n}=1+d n \beta^{-1} t^{w_{i_{4}}}+*$ for all $n \in \mathbb{N}$.

Let $J=\left\{i_{1}, i_{2}, i_{3}, i_{4}\right\}$. Using Lemma B.5. $\operatorname{val}\left(D_{J}(b)\right)=w_{i_{4}}$.

To prove that there exists $f=\sum_{i=0}^{n} c_{i} x^{i}$ with double roots 1 and $b$ in $S e v_{n}^{2} \cap \mathbb{K}[x]_{w}$, we need to see that there exist a vector of coefficients $\left(c_{i}\right)_{i \in \mathcal{A}}$ that satisfies Equation (4). As the minor $D_{J}(b)$ is not zero, there exists a unique vector of solutions $c=\left(c_{i_{1}}, c_{i_{2}}, c_{i_{3}}, c_{i_{4}}\right) \in \mathbb{K}^{4}$ obtained by taking $c_{i}=t^{w_{i}}$ for all $i \notin J$. It only remains to be seen that $\operatorname{val}\left(c_{i_{j}}\right)=0$ for all $1 \leq j \leq 3$ and $\operatorname{val}\left(c_{i_{4}}\right)=w_{i_{4}}$.

Let $S$ be the set of all $s \in \mathcal{A}$ such that $s \equiv i_{1} \bmod d$ and $0<\operatorname{val}\left(w_{s}\right) \leq w_{i_{4}}$. In particular, because of the second defining condition on $S$ we have $i_{1}, i_{2}, i_{3} \notin S$. As $w$ is generic, we can assume that either $S=\emptyset$ or $\left\{s \in S: w_{s} \leq w_{k}\right.$ for all $k \in$ $S\}=\left\{s_{0}\right\}$. We re-write the system of Equation (4) as

$$
\mathbf{M}_{J}(b) \cdot c^{T}=-\mathbf{M}_{S}(b) \cdot\left(t^{w_{s}}\right)_{s \in S}^{T}-\mathbf{M}_{\mathcal{A}-(S \cup J)}(b)\left(t^{w_{j}}\right)_{j \notin S \cup I}^{T},
$$

we are going to find the valuation of the solutions $c^{(1)}=\left(c_{i_{j}}^{(1)}\right)_{j=1}^{4}$ and $c^{(2)}=$ $\left(c_{i_{j}}^{(2)}\right)_{j=1}^{4}$ for the following associate systems:

$$
\begin{aligned}
\mathbf{M}_{J}(b) \cdot\left(c^{(1)}\right)^{T}= & -\mathbf{M}_{S}(b) \cdot\left(t^{w_{s}}\right)_{s \in S}^{T}=-\left(t^{w_{s_{0}}}, s_{0} t^{w_{s_{0}}}, b^{s_{0}} t^{w_{s_{0}}}, s_{0} b^{s_{0}} t^{w_{s_{0}}}\right)^{T}, \\
\mathbf{M}_{J}(b) \cdot\left(c^{(2)}\right)^{T} & =-\mathbf{M}_{\mathcal{A}-(S \cup J)}(b) \cdot\left(t^{w_{j}}\right)_{j \notin S \cup I}^{T} \\
& =-\left(t^{w_{i_{5}}}+*, i_{5} t^{w_{i_{5}}}+*, b^{i_{5}} t^{w_{i_{5}}}+*, i_{5} b^{i_{5}} t^{w_{i_{5}}}+*\right)^{T} .
\end{aligned}
$$

We denote by $\operatorname{det}\left(\mathbf{M}_{J^{\prime}}(b) \mid C\right)$ the determinant of the matrix in $\mathbb{K}^{4 \times 4}$ where the first columns are given by the matrix $\mathbf{M}_{J^{\prime}}(b)$ (for some set $J^{\prime}$ of cardinal 3) and the last one is a fixed column $C$. In a similar way $\operatorname{det}\left(C \mid \mathbf{M}_{J^{\prime}}(b)\right)$ is the determinant of the matrix $\mathbf{M}_{J^{\prime}}(b)$ extended with a first column $C$.

First, if $S \neq 0$, we solve Equation (7). Consider the case of $c_{i_{1}}^{(1)}\left(\right.$ as $s_{0} \equiv i_{1} \bmod d$ we can use Lemma B.5 and we have, for $C=\left(t^{w_{s_{0}}}, s_{0} t^{w_{s_{0}}}, b^{s_{0}} t^{w_{s_{0}}}, s_{0} b^{s_{0}} t^{w_{s_{0}}}\right)$, that

$$
\operatorname{det}\left(C \mid \mathbf{M}_{\left\{i_{2}, i_{3}, i_{4}\right\}}(b)\right)=t^{w_{s_{0}}} \operatorname{det}\left(\mathbf{M}_{\left\{s_{0}, i_{2}, i_{3}, i_{4}\right\}}(b)\right)
$$


has valuation $w_{s_{0}}+w_{i_{4}}$. Therefore, by Cramer's rule

$$
\operatorname{val}\left(c_{i_{1}}^{(1)}\right)=w_{s_{0}}+w_{i_{4}}-w_{i_{4}}=w_{s_{0}}>0 .
$$

As $i_{1} \equiv i_{2} \equiv i_{3} \bmod d$, we can prove equivalently that $\operatorname{val}\left(c_{i_{2}}^{(1)}\right)=w_{s_{0}}>0$ and $\operatorname{val}\left(c_{i_{3}}^{(1)}\right)=w_{s_{0}}>0$. The case of $c_{i_{4}}$ is slightly different because we are replacing the fourth column and $s_{0} \equiv i_{j} \bmod d$ for all $1 \leq j \leq 3$. Using again Lemma B.5 and $C=\left(t^{w_{s_{0}}}, s_{0} t^{w_{s_{0}}}, b^{s_{0}} t^{w_{s_{0}}}, s_{0} b^{s_{0}} t^{w_{s_{0}}}\right)$, the valuation $\operatorname{val}\left(\operatorname{det}\left(\mathbf{M}_{\left\{i_{1}, i_{2}, i_{3}\right\}}(b) \mid C\right)\right)=$ $w_{s_{0}}+4 w_{i_{4}}$, and therefore $\operatorname{val}\left(c_{i_{4}}^{(1)}\right) \geq w_{s_{0}}+4 w_{i_{4}}-w_{i_{4}}>w_{i_{4}}$.

Consider now the case of $c_{i_{1}}^{(2)}$, we have to take into account that $i_{5} \not \equiv i_{1} \bmod d$. Then, considering the vector $C=\left(t^{w_{i_{5}}}+*, i_{5} t^{w_{i_{5}}}+*, b^{i_{5}} t^{w_{i_{5}}}+*, i_{5} b^{i_{5}} t^{w_{i_{5}}}+*\right)$,

$$
\operatorname{det}\left(C \mid \mathbf{M}_{\left\{i_{2}, i_{3}, i_{4}\right\}}(b)\right)=t^{w_{i_{5}}} \beta^{i_{5}+i_{2}}\left(i_{3}-i_{2}\right)\left(i_{5}-i_{4}\right)\left(\beta^{i_{2}-i_{5}}-1\right)\left(\beta^{i_{4}-i_{2}}-1\right)+*
$$

has valuation $w_{i_{5}}=w_{i_{4}}$, and hence $\operatorname{val}\left(c_{i_{1}}^{(2)}\right)=0$. Again, as $i_{1} \equiv i_{2} \equiv i_{3} \bmod d$ we have $\operatorname{val}\left(c_{i_{2}}^{(2)}\right)=0$ and $\operatorname{val}\left(c_{i_{3}}^{(2)}\right)=0$. For the case of $c_{i_{4}}^{(2)}$, we can find the valuation similarly to Lemma B.5. Taking $C=\left(t^{w_{i_{5}}}+*, i_{5} t^{w_{i_{5}}}+*, b^{i_{5}} t^{w_{i_{5}}}+*, i_{5} b^{i_{5}} t^{w_{i_{5}}}+*\right)$, we obtain

$\operatorname{det}\left(\mathbf{M}_{\left\{i_{1}, i_{2}, i_{3}\right\}}(b) \mid C\right)=-t^{w_{i_{5}}+w_{i_{4}}} \beta^{2 i_{1}-1}\left(i_{3}-i_{2}\right)\left(i_{3}-i_{1}\right)\left(i_{2}-i_{1}\right)\left(\beta^{i_{5}-i_{1}}-1\right)+*$.

Hence $\operatorname{val}\left(c_{i_{4}}^{(2)}\right)=w_{i_{5}}+w_{i_{4}}-w_{i_{4}}=w_{i_{4}}$.

Taking $c=\left(c_{i_{1}}, c_{i_{2}}, c_{i_{3}}, c_{i_{4}}\right)=\left(c_{i_{1}}^{(1)}+c_{i_{1}}^{(2)}, \ldots, c_{i_{4}}^{(1)}+c_{i_{4}}^{(2)}\right)$, this is the solution for Equation 6 . As $\operatorname{val}\left(c_{i_{j}}^{(1)}\right)>0$ and $\operatorname{val}\left(c_{i_{j}}^{(2)}\right)=0$ for all $1 \leq j \leq 3, \operatorname{val}\left(c_{i_{4}}^{(1)}\right)>w_{i_{4}}$ and $\operatorname{val}\left(c_{i_{4}}^{(2)}\right)=w_{i_{4}}$, then every coordinate of $c$ satisfies $\operatorname{val}\left(c_{i_{j}}\right)=w_{i_{j}}$ for all $1 \leq$ $j \leq 4$ as wanted.

\section{Appendix A. Proof of Lemma 2.2}

We give here the proof of Lemma 2.2 , that asserts that the tropicalization of a linear homogeneous ideal $I$ over a valuated field $\mathbb{K}$ with infinite residue field is determined by the circuits.

Proof. We denote by $L(I)$ the intersection $\bigcap_{\ell \in I: \ell \text { linear form }} V(\operatorname{trop}(\ell))$. Let us first prove the equality $\mathcal{T}(I)=L(I)$. Clearly, $\mathcal{T}(I) \subseteq L(I)$. We will see the other inclusion by induction on the codimension of $V=V(I) \cap\left(\mathbb{K}^{*}\right)^{n}$.

Note that $V=\emptyset$ if and only if $V(I)$ is contained in a hyperplane $x_{i}=0$ and so $x_{i} \in I$ and $\mathcal{T}(I)=L(I)=\emptyset$. Hence, we can assume that $V \neq \emptyset$.

If $\operatorname{codim}(V)=1$, then $V$ is a hyperplane and there exists a linear form $\ell$ such that $V=V(\ell) \cap\left(\mathbb{K}^{*}\right)^{n}$. In this case is clear that $\mathcal{T}(I)=V(\operatorname{trop}(\ell))$. If the result is true for every codimension less than or equal to $r$, let us take $V$ of codimension $r+1$. Let $p \in L(I)$. If there exists $e_{i}$ in the canonical basis such that $e_{i} \in V(I)$ for some $i \in\{1, \ldots, n\}$, then for all $\ell \in I$ linear form, $\ell(x)=\sum_{j=1}^{n} \ell_{j} x_{j}$ with $\ell_{i}=0$. Let $\pi_{i}: \mathbb{K}^{n} \longrightarrow \mathbb{K}^{n-1}$ be the projection $\pi_{i}\left(x_{1}, \ldots, x_{n}\right)=\left(x_{1}, \ldots, x_{i-1}, x_{i+1}, \ldots, x_{n}\right)$. Then, $p \in \mathcal{T}(I)$ if and only if $\pi_{i}(p) \in \mathcal{T}\left(\pi_{i}(V)\right)$ and the result follows by induction in the codimension.

If $e_{i} \notin V(I)$ for all $1 \leq i \leq n, V(I) \subsetneq V(I) \oplus\left\langle e_{i}\right\rangle$ for all $i$. By our inductive hypothesis, $\mathcal{T}\left(V(I) \oplus\left\langle e_{i}\right\rangle\right)$ equals the intersection of $V(\operatorname{trop}(\ell))$, for all linear forms $\ell$ with $V(I) \oplus\left\langle e_{i}\right\rangle \subseteq\{\ell=0\}$. Therefore, $p \in \bigcap_{i=1}^{n} \mathcal{T}\left(V(I) \oplus\left\langle e_{i}\right\rangle\right)$. Without loss of generality we can assume $p=(0, \ldots, 0)$. Hence, for each $1 \leq i \leq n$ there is a point $u_{i}=\left(u_{i 1}, \ldots, u_{i n}\right) \in V(I)$ such that for all $i \neq j, \operatorname{val}\left(u_{i j}\right)=0$. As $\operatorname{codim}(V)>1$, the matrix $U$ with $U_{i j}=u_{i j}$ for all $1 \leq i, j \leq n$ has rank at most $n-2$. Then, $\operatorname{det}(U)=0$, and so there exist $i \in\{1, \ldots, n\}$ such that $\operatorname{val}\left(u_{i i}\right) \geq 0$. Since the minor obtained from the matrix $U$ deleting the $i$-th row and column is 
also singular, there must be another element $j \in\{0, \ldots, n\}-\{i\}$ with $\operatorname{val}\left(u_{j j}\right) \geq 0$. Without loss of generality, let $\{i, j\}=\{1,2\}$. If $\operatorname{val}\left(u_{11}\right)=0$ or $\operatorname{val}\left(u_{22}\right)=0$, then $p=\operatorname{val}\left(u_{1}\right) \in \mathcal{T}(I)$ or $p=\operatorname{val}\left(u_{2}\right) \in \mathcal{T}(I)$. If not, we can take a generic $\lambda \in \mathbb{K}^{*}$ of valuation zero such that $\operatorname{trop}\left(u_{1}+\lambda u_{2}\right)=p$ and therefore $p \in \mathcal{T}(I)$. In fact, let $\lambda$ be generic so $\operatorname{val}\left(u_{1}+\lambda u_{2}\right)_{i}=0$ for all $3 \leq i \leq n$. As $\operatorname{val}\left(u_{11}\right)>0$ and $\operatorname{val}\left(\lambda u_{21}\right)=0$, then $\operatorname{val}\left(u_{11}+\lambda u_{21}\right)=0$. Similarly $\operatorname{val}\left(u_{12}+\lambda u_{22}\right)=0$.

To see that it is enough to consider the circuits in $I$ to describe $\mathcal{T}(I)$, recall that, for each minimal support, there is only (up to multiplication by a constant) one linear form in $I$. Let us call $\left\{\ell_{1}^{\prime}, \ldots, \ell_{r}^{\prime}\right\}$ linear forms generating $I$. Clearly, $\mathcal{T}(I) \subseteq \cap_{i=1}^{r} V\left(\operatorname{trop}\left(\ell_{i}^{\prime}\right)\right)$. Given $p \notin \mathcal{T}(I)$, there is a linear form $\ell \in I$ such that $p \notin V(\operatorname{trop}(\ell))$. It suffices to check that we can take $\ell$ with minimal support. Let $\ell_{1}:=\ell$. If $\ell_{1}$ does not have minimal support, then there exists a linear form $g_{1} \in I$ whose support is contained in the support of $\ell_{1}$. If $p \notin V\left(\operatorname{trop}\left(g_{1}\right)\right)$, we are done. If $p \in V\left(\operatorname{trop}\left(g_{1}\right)\right)$, let $x_{m_{1}}$ and $x_{m_{2}}$ be different variables such that the associated linear forms in $\operatorname{trop}\left(\ell_{1}\right)$ and $\operatorname{trop}\left(g_{1}\right)$ attain respectively the minimum at $p$. We can take $\ell_{2}$ a linear combination of $\ell_{1}$ and $g_{1}$ to make zero the monomial $x_{m_{2}}$ in $\ell_{2}$ without modifying where $\operatorname{trop}\left(\ell_{2}\right)$ attains its minimum, and proceed recursively.

\section{Appendix B. The MAtrix M AND its Minors}

In this section, we prove all the claims about the matrix $\mathbf{M}$ in Definition 3.1 its roots, rank etc. used in the paper. First of all, by the following lemma we can assume (when needed, to simplify the notation) that $i_{1}=0$.

Lemma B.1. With the previous notation, the minor $D_{J}$ is invariant by translations of $i_{1}, i_{2}, i_{3}$ and $i_{4}$ (up to a monomial factor). More specifically, for $s \in \mathbb{N}$

$$
x^{2 s} D_{J}(x)=D_{J(+s)}(x) \text { and } D_{J(\times s)}(x)=s^{2} \cdot D_{J}\left(x^{s}\right)
$$

Also, given positive $i<j<k$,

$D_{\{0, i, j, k\}}(x)=i k x^{i}\left(x^{k-i}-1\right)\left(x^{j}-1\right)-i j x^{i}\left(x^{j-i}-1\right)\left(x^{k}-1\right)-j k x^{j}\left(x^{k-j}-1\right)\left(x^{i}-1\right)$, and we have the equality

$$
D_{\{0, i, j, k\}}(x)=x^{i+j+k} D_{\{0, i, j, k\}}\left(x^{-1}\right) .
$$

Proof. It is a straightforward computation from the properties of the determinant.

Lemma B.2. Let $J=\left\{i_{1}, i_{2}, i_{3}, i_{4}\right\}$ be a subset of $\mathcal{A}$ with $i_{1}<i_{2}<i_{3}<i_{4}$. Then $D_{J}$ is not the zero polynomial, its degree (as a polynomial in $x$ ) is $i_{3}+i_{4}$ and its order at the origin equals $i_{1}+i_{2}$. Moreover, the matrix $\mathbf{M}(x)$ has rank 4 if $x \neq 0,1$ and $n \geq 3$.

Proof. By Lemma B.1.

$$
D_{J}(x)=x^{2 i_{1}} D_{\left\{0, i_{2}-i_{1}, i_{3}-i_{1}, i_{4}-i_{1}\right\}}=\left(i_{2}-i_{1}\right)\left(i_{4}-i_{3}\right)\left(x^{i_{3}+i_{4}}+\cdots+x^{i_{1}+i_{2}}\right) .
$$

If we consider the submatrix $\mathbf{M}_{J} \in(\mathbb{Z}[x])^{4 \times 4}$ given by $J=\{0,1,2,3\}$, it holds that $D_{J}=x(x-1)^{4}$, and hence $\operatorname{rank}(\mathbf{M}(x))=4$ for all $x \neq 0,1$.

Lemma B.3. Let $J=\left\{i_{1}, i_{2}, i_{3}, i_{4}\right\}$ be a subset of $\mathcal{A}$ such that $i_{1}<i_{2}<i_{3}<i_{4}$. Let $\beta \in K$. Then, the matrix $\mathbf{M}_{J}(\beta)$ has rank 2 if and only if $\beta^{i_{1}}=\beta^{i_{2}}=\beta^{i_{3}}=\beta^{i_{4}}$.

Proof. We can assume $\beta \neq 0$ since the result is trivial otherwise. It is clear that $\beta^{i_{1}}=\beta^{i_{2}}=\beta^{i_{3}}=\beta^{i_{4}}$ is a sufficient condition for $\operatorname{rank}\left(\mathbf{M}_{J}(\beta)\right)=2$. To see that it is necessary, using Lemma B.1 we can assume $i_{1}=0$. Let us note that if the matrix $\mathbf{M}_{J}(\beta)$ has rank 2 , then all the $3 \times 3$ minors are zero including $\operatorname{det}(\mathbf{M}(4,4))=$ $i_{2}\left(\beta^{i_{3}}-1\right)-i_{3}\left(\beta^{i_{2}}-1\right), \operatorname{det}(\mathbf{M}(3,4))=i_{2} i_{3}\left(\beta^{i_{3}}-\beta^{i_{2}}\right)$ and $\operatorname{det}(\mathbf{M}(3,3)) i_{2} i_{4}\left(\beta^{i_{4}}-\beta^{i_{2}}\right)$, where $\mathbf{M}(i, j)$ is the submatrix obtained by removing the row $i$ and column $j$ from 
$M_{J}(\beta)$. From the last two, $\beta^{i_{3}}=\beta^{i_{2}}=\beta^{i_{4}}$ and using this in the the first minor we have $\beta^{i_{2}}=1=\beta^{i_{1}}$.

Lemma B.4. Let $J=\left\{i_{1}, i_{2}, i_{3}, i_{4}\right\}$ be a subset of $\mathcal{A}$ such that $i_{1}<i_{2}<i_{3}<i_{4}$ and $\beta \in K$ a root of unity. Consider the powers $\beta^{i_{1}}, \ldots, \beta^{i_{4}}$ of $\beta$. If all four are equal (e.g. $\beta=1$ ), then $\beta$ is a root of $D_{J}$ of multiplicity 4 . If exactly three of the powers are equal and the remaining one is different, then the multiplicity of $\beta$ is 1 .

Proof. For the multiplicity of $\beta$ when all the powers of $\beta$ are equal, assuming without loss of generality that $i_{1}>3$, the first 4 derivatives of $D_{J}$ can be calculated to see that $D_{J}(\beta)=D_{J}^{\prime}(\beta)=D_{J}^{(2)}(\beta)=D_{J}^{(3)}(\beta)=0$ while $D_{J}^{(4)}(\beta)=$ $2\left(i_{1}-i_{2}\right)\left(i_{1}-i_{3}\right)\left(i_{1}-i_{4}\right)\left(i_{2}-i_{3}\right)\left(i_{2}-i_{4}\right)\left(i_{3}-i_{4}\right) \beta^{2 i_{1}-4} \neq 0$.

When only three of the powers of $\beta$ are equal, we can assume $\beta^{i_{1}} \neq \beta^{i_{2}}=\beta^{i_{3}}=$ $\beta^{i_{4}}$. Then it is clear that $D_{J}(\beta)=0$, but $D_{J}^{\prime}(\beta)=\beta^{i_{2}-1}\left(\beta^{i_{1}}-\beta^{i_{2}}\right)\left(i_{4}-i_{3}\right)\left(i_{4}-\right.$ $\left.i_{2}\right)\left(i_{3}-i_{2}\right) \neq 0$

Lemma B.5. Let $J=\left\{i_{1}, i_{2}, i_{3}, i_{4}\right\} \subset \mathcal{A}$ such that $g=\operatorname{gcd}\left(i_{3}-i_{1}, i_{2}-i_{1}\right)>1$ and $\# J=4$. Let $\beta \in K \backslash\{1\}$ be a root of unity such that $\beta^{g}=1$. Consider $b=\beta+h t^{v}+* \in \mathbb{K}$, where $h \in K \backslash\{0\}$ and $v>0$. Then, the valuation of $D_{J}(b)$ is $4 v$ if $\beta^{i_{4}-i_{1}}=1$, and $v$ otherwise.

Proof. To compute $D_{J}(b)$ (up to a sign) we can assume $i_{1}<i_{2}<i_{3}$ and use the $i_{4}$-th column of $\mathbf{M}$ as the last one of $D_{J}$. By Lemma B.1 and the facts that $b \neq 0$ and $\operatorname{val}\left(b^{2 i_{1}}\right)=0$ we can assume $i_{1}=0$. Also, by the formulation of $D_{J}$ in the same Lemma (where now $i_{4}$ is possibly a negative number),

$D_{J}(b)=i_{2} i_{4} b^{i_{2}}\left(b^{i_{4}-i_{2}}-1\right)\left(b^{i_{3}}-1\right)-i_{2} i_{3} b^{i_{2}}\left(b^{i_{3}-i_{2}}-1\right)\left(b^{i_{4}}-1\right)-i_{3} i_{4} b^{i_{3}}\left(b^{i_{4}-i_{3}}-1\right)\left(b^{i_{2}}-1\right)$,

Let $d>1$ be the order of $\beta$ as a root of unity. If $d \nmid l, b^{l}-1=\beta^{l}-1+*$ of valuation 0 , while if $d \mid l, b^{l}=1+*$ of valuation 0 and $b^{l}-1=l \beta^{-1} h t^{v}+*$ of valuation $v$. If $d \nmid i_{4}, D_{J}(b)=-\beta^{-1} h i_{2} i_{3}\left(i_{3}-i_{2}\right)\left(\beta^{i_{4}}-1\right) t^{v}+*$ which has valuation $v$.

In case that $d \mid i_{4}$, let $d^{\prime}:=\operatorname{gcd}\left(i_{4}, i_{3}, i_{2}\right)$. Note that $d \mid d^{\prime}$. Using the notation $i_{j}^{\prime}=\frac{i_{j}}{d^{\prime}}$ and $J^{\prime}=\left\{0, i_{2}^{\prime}, i_{3}^{\prime}, i_{4}^{\prime}\right\}$, we have by Lemma B.1

$$
D_{J}(b)=D_{J^{\prime}}\left(b^{d^{\prime}}\right)=D_{J^{\prime}}\left(1+d^{\prime} \beta^{-1} h t^{v}+*\right) \text {. }
$$

By Lemma B.4, the multiplicity of 1 as a root of $D_{J^{\prime}}$ is 4 , that is there exists a polynomial $p \in \mathbb{Z}[x]$ such that $p(1) \neq 0$ and $D_{J^{\prime}}(x)=(x-1)^{4} p(x)$. Then, $D_{J}(b)=\left(d^{\prime} \beta^{-1} h t^{v}+*\right)^{4} p\left(1+d^{\prime} \beta^{-1} h t^{v}+*\right)$, which has valuation $4 v$.

Lemma B.6. Let $\beta \in K \backslash\{0\}$ and $J=\left\{i_{1}, i_{2}, i_{3}, i_{4}, i_{5}\right\}$ be a subset of $\mathcal{A}$ of cardinal 5 such that $D_{J-\left\{i_{5}\right\}}(\beta)=0$ and the set $\left\{\beta^{i_{1}}, \beta^{i_{2}}, \beta^{i_{3}}, \beta^{i_{4}}\right\}$ does not have three elements equal and the remaining one different. Then, either $D_{J-\left\{i_{j}\right\}}(\beta)=0$ for all $1 \leq j \leq 5$, or $D_{J-\left\{i_{j}\right\}}(\beta) \neq 0$ for all $1 \leq j \leq 4$.

Proof. Since we are not assuming that the indices $\left\{i_{j}\right\}_{i=1}^{4}$ are ordered, it is enough to prove that if $D_{J-\left\{i_{1}\right\}}(\beta)=0$ then $D_{J-\left\{i_{j}\right\}}(\beta)=0$ for all $2 \leq j \leq 4$. This result follows from the direct computation below.

Let $y_{1}, y_{2}, y_{3}, y_{4}, y_{5}, x_{1}, x_{2}, x_{3}, x_{4}, x_{5}$ be variables over $K$ and $\mathcal{J}_{1}$ the ideal generated by the $4 \times 4$ minors of the matrix

$$
M=\left(\begin{array}{ccccc}
1 & 1 & 1 & 1 & 1 \\
y_{1} & y_{2} & y_{3} & y_{4} & y_{5} \\
x_{1} & x_{2} & x_{3} & x_{4} & x_{5} \\
y_{1} x_{1} & y_{2} x_{2} & y_{3} x_{3} & y_{4} x_{4} & y_{5} x_{5}
\end{array}\right) .
$$

Let $f$ be the polynomial $f\left(y_{2}, y_{3}, y_{4}\right)=\left(y_{4}-y_{3}\right)\left(y_{4}-y_{2}\right)\left(y_{3}-y_{2}\right)$ and $\mathcal{I}_{2}, \mathcal{I}_{3}$ be the ideals $\mathcal{I}_{2}=\left\langle\operatorname{det}\left(M_{\{1,2,3,4\}}\right), \operatorname{det}\left(M_{\{2,3,4,5\}}\right)\right\rangle, \mathcal{I}_{3}=\left\langle x_{2}-x_{3}, x_{2}-x_{4}\right\rangle$. Then, by direct computation (see Computation C.2 we can check that the ideals $\left(\mathcal{I}_{2}: f\right)$ and $\mathcal{I}_{1} \cap \mathcal{I}_{3}$ are equal. 
If $D_{J-\left\{i_{5}\right\}}(\beta)=D_{J-\left\{i_{1}\right\}}(\beta)=0$, then $\left(i_{1}, \ldots, i_{5}, \beta^{i_{1}}, \ldots, \beta^{i_{5}}\right)$ is in the variety defined by $\mathcal{I}_{2}$ and is not a root of $f$. Then is in the variety defined by $\left(\mathcal{I}_{2}: f\right)$ and therefore gives us two choices:

- If the point $\left(i_{1}, \ldots, i_{5}, \beta^{i_{1}}, \ldots, \beta^{i_{5}}\right)$ is in the variety defined by $\mathcal{I}_{1}$, then $D_{J-\left\{i_{j}\right\}}(\beta)=0$ for all $1 \leq j \leq 5$.

- If the point $\left(i_{1}, \ldots, i_{5}, \beta^{i_{1}}, \ldots, \beta^{i_{5}}\right)$ is in the variety defined by $\mathcal{I}_{3}$, then $\beta^{i_{2}}=\beta^{i_{3}}=\beta^{i_{4}}$. By the hypothesis of the lemma, these three powers have to be equal to $\beta^{i_{1}}$ and hence $\operatorname{rank}\left(M_{J-\left\{i_{5}\right\}}\left(i_{1}, \ldots, i_{5}, \beta^{i_{1}}, \ldots, \beta^{i_{5}}\right)\right)=2$. Thus it is also true that $D_{J-\left\{i_{j}\right\}}(\beta)=0$ for all $1 \leq j \leq 5$.

Lemma B.7. Let $J=\left\{i_{1}, i_{2}, i_{3}, i_{4}\right\}$ be a subset of $\mathcal{A}$ and $\beta \in K \backslash\{0\}$ a root of $D_{J}$ of multiplicity at least 3 . Then $\beta$ is a root of unity and $\beta^{i_{1}}=\beta^{i_{2}}=\beta^{i_{3}}=\beta^{i_{4}}$.

Proof. Let $y_{1}, y_{2}, y_{3}, y_{4}, x_{1}, x_{2}, x_{3}, x_{4}$ be variables over $K$ and $\mathcal{I}$ the ideal generated by $\operatorname{det}(M), \operatorname{det}\left(M_{1}\right)$ and $\operatorname{det}\left(M_{21}\right)+\operatorname{det}\left(M_{22}\right)$, where

$$
\begin{aligned}
& M=\left(\begin{array}{cccc}
1 & 1 & 1 & 1 \\
y_{1} & y_{2} & y_{3} & y_{4} \\
x_{1} & x_{2} & x_{3} & x_{4} \\
y_{1} x_{1} & y_{2} x_{2} & y_{3} x_{3} & y_{4} x_{4}
\end{array}\right), M_{1}=\left(\begin{array}{cccc}
1 & 1 & 1 & 1 \\
y_{1} & y_{2} & y_{3} & y_{4} \\
x_{1} & x_{2} & x_{3} & x_{4} \\
y_{1}^{2} x_{1} & y_{2}^{2} x_{2} & y_{3}^{2} x_{3} & y_{4}^{2} x_{4}
\end{array}\right), \\
& M_{21}=\left(\begin{array}{cccc}
1 & 1 & 1 & 1 \\
y_{1} & y_{2} & y_{3} & y_{4} \\
x_{1} & x_{2} & x_{3} & x_{4} \\
y_{1}^{2}\left(y_{1}-1\right) x_{1} & y_{2}^{2}\left(y_{2}-1\right) x_{2} & y_{3}^{2}\left(y_{3}-1\right) x_{3} & y_{4}^{2}\left(y_{4}-1\right) x_{4}
\end{array}\right) \text { and } \\
& M_{22}=\left(\begin{array}{cccc}
1 & 1 & 1 & 1 \\
y_{1} & y_{2} & y_{3} & y_{4} \\
y_{1} x_{1} & y_{2} x_{2} & y_{3} x_{3} & y_{4} x_{4} \\
y_{1}^{2} x_{1} & y_{2}^{2} x_{2} & y_{3}^{2} x_{3} & y_{4}^{2} x_{4}
\end{array}\right) \text {. }
\end{aligned}
$$

Note that when we evaluate the matrices in $y_{j}=i_{j}, x_{j}=x^{i_{j}}$, then $\operatorname{det}\left(M_{1}\right)(x)=$ $\frac{\partial}{\partial x} \operatorname{det}(M)(x)$ and $\operatorname{det}\left(M_{21}\right)(x)+\operatorname{det}\left(M_{22}\right)(x)=\frac{\partial^{2}}{\partial x^{2}} \operatorname{det}(M)(x)$, so $\mathcal{I}$ is the ideal where $D_{J}$ and its two derivatives vanish.

Let $f, g$ be the polynomials $f\left(y_{1}, y_{2}, y_{3}, y_{4}\right)=\prod_{1<j<k<4}\left(y_{k}-y_{j}\right), g\left(x_{1}, x_{2}, x_{3}, x_{4}\right)=$ $x_{1} x_{2} x_{3} x_{4}$ and $\mathcal{I}_{1}=\left(\mathcal{I}: f^{\infty}\right)$. Then, by direct computation (See Computation C.3 we can check that the ideal $\mathcal{I}_{2}=\left(\sqrt{\mathcal{I}_{1}}: g\right)$ is exactly $\left\langle x_{4}-x_{3}, x_{3}-x_{2}, x_{2}-x_{1}\right\rangle$. If $\beta$ is at least a triple root of $D_{J}$, as $\left(i_{1}, i_{2}, i_{3}, i_{4}, \beta^{i_{1}}, \beta^{i_{2}}, \beta^{i_{3}}, \beta^{i_{4}}\right)$ is not a root of $f$ and $\beta \neq 0$, then is in the variety defined by $\mathcal{I}_{2}$ and hence $\beta$ is a root of unity with $\beta^{i_{1}}=\beta^{i_{2}}=\beta^{i_{3}}=\beta^{i_{4}}$.

Lemma B.8. Let $J=\left\{i_{1}, i_{2}, i_{3}, i_{4}, i_{5}\right\}$ be a subset of $\mathcal{A}$ of cardinal 5 and $\beta \in$ $K \backslash\{0\}$ a common root of $D_{J-\left\{i_{j}\right\}}$ for all $1 \leq j \leq 5$ such that the elements in $\left\{\beta^{i_{j}}\right\}_{j=1}^{4}$ are repeated at most once. If $\beta$ is a multiple root of $D_{J-\left\{i_{5}\right\}}$, then it is a multiple root of $D_{J-\left\{i_{j}\right\}}$ for all $1 \leq j \leq 4$.

Proof. Without loss of generality we can assume $\beta^{i_{1}} \neq \beta^{i_{2}}$ and $\beta^{i_{1}} \neq \beta^{i_{3}}$.

Let $y_{1}, y_{2}, y_{3}, y_{4}, y_{5}, x_{1}, x_{2}, x_{3}, x_{4}, x_{5}$ be variables over $K$ and $\mathcal{I}$ the ideal generated by the $4 \times 4$ minors of the matrix $M$ and $\operatorname{det}\left(\left(M_{1}\right)_{\{1,2,3,4\}}\right)$ where

$$
M=\left(\begin{array}{ccccc}
1 & 1 & 1 & 1 & 1 \\
y_{1} & y_{2} & y_{3} & y_{4} & y_{5} \\
x_{1} & x_{2} & x_{3} & x_{4} & x_{5} \\
y_{1} x_{1} & y_{2} x_{2} & y_{3} x_{3} & y_{4} x_{4} & y_{5} x_{5}
\end{array}\right), M_{1}=\left(\begin{array}{ccccc}
1 & 1 & 1 & 1 & 1 \\
y_{1} & y_{2} & y_{3} & y_{4} & y_{5} \\
x_{1} & x_{2} & x_{3} & x_{4} & x_{5} \\
y_{1}^{2} x_{1} & y_{2}^{2} x_{2} & y_{3}^{2} x_{3} & y_{4}^{2} x_{4} & y_{5}^{2} x_{5}
\end{array}\right) .
$$

Let $f$ be the polynomial $f\left(y_{1}, y_{2}, y_{3}, y_{4}\right)=\prod_{1 \leq j<k \leq 4}\left(y_{k}-y_{j}\right), \mathcal{I}_{1}=\left\langle x_{1}-x_{2}, x_{1}-x_{3}\right\rangle$ and $\mathcal{I}_{2}=\left(\mathcal{I}: \mathcal{I}_{1}\right)$. Then, by direct computation (see Computation C.4) we can 
check that the $4 \times 4$ minors of the matrix $M_{1}$ are elements of the ideal $\mathcal{I}_{3}=\left(\mathcal{I}_{2}: f^{\infty}\right)$. If $\beta$ is a common root of $D_{J-\left\{i_{j}\right\}}$ for all $1 \leq j \leq 4$ and a multiple root of $D_{J-\left\{i_{5}\right\}}$, then $\left(i_{1}, i_{2}, i_{3}, i_{4}, i_{5}, \beta^{i_{1}}, \beta^{i_{2}}, \beta^{i_{3}}, \beta^{i_{4}}, \beta^{i_{5}}\right)$ is in the variety defined by $\mathcal{I}_{3}$ and hence $\beta$ is a multiple root of $D_{J-\left\{i_{j}\right\}}$ for all $1 \leq j \leq 4$.

Lemma B.9. Let $J=\left\{i_{1}, i_{2}, i_{3}, i_{4}\right\}$ be a subset of $\mathcal{A}$ and $\beta \in K \backslash\{0,1\}$ a root of $D_{J}$ such that the powers in $\left\{\beta^{i_{j}}\right\}_{j=1}^{4}$ are repeated at most once. Then there exists $i_{5} \in \mathcal{A}-J$ such that $D_{\left(J \cup\left\{i_{5}\right\}\right)-\left\{i_{j}\right\}}$ does not vanish at $\beta$ for all $1 \leq j \leq 4$.

Proof. Note that if $\beta$ is a root of $D_{\left(J \cup\left\{i_{5}\right\}\right)-\left\{i_{1}\right\}}$, then by Lemma B.6 $\beta$ it is a root of every $D_{\left(J \cup\left\{i_{5}\right\}\right)-\left\{i_{j}\right\}}$ for all $1 \leq j \leq 4$. We now see this can not happen for every $i_{5} \in\left\{i_{1}, i_{1}+1, i_{1}+2, i_{1}+3\right\}$.

Let $y_{1}, y_{2}, y_{3}, y_{4}, x_{1}, x_{2}, x_{3}, x_{4}, x$ be variables over $K$ and $\mathcal{I}$ the ideal generated by the $4 \times 4$ minors of the matrices $M_{i}$ for all $0 \leq i \leq 3$ where

$$
M_{i}=\left(\begin{array}{ccccc}
1 & 1 & 1 & 1 & 1 \\
y_{1} & y_{2} & y_{3} & y_{4} & y_{1}+i \\
x_{1} & x_{2} & x_{3} & x_{4} & x_{1} x^{i} \\
y_{1} x_{1} & y_{2} x_{2} & y_{3} x_{3} & y_{4} x_{4} & \left(y_{1}+i\right) x_{1} x^{i}
\end{array}\right) .
$$

Let $f$ be the polynomial $f\left(y_{1}, y_{2}, y_{3}, y_{4}\right)=\prod_{1 \leq j<k \leq 4}\left(y_{k}-y_{j}\right)$. By direct computation (see Computation C.5 we can check the equality of ideals $\left(\mathcal{I}: x_{1} x_{4} x(x-1)^{4} f\right)=$ $\left\langle x_{1}-x_{4}, x_{2}-x_{4}, x_{3}-x_{4}\right\rangle$. If $D_{\left(J \cup\left\{i_{1}+i\right\}\right)-\left\{i_{j}\right\}}(\beta)=0$ for all $1 \leq j \leq 4$ and $0 \leq i \leq 3$, then $\left(i_{1}, \ldots, i_{4}, \beta^{i_{1}}, \ldots, \beta^{i_{4}}, \beta\right)$ is in the variety defined by $\mathcal{I}$ but is not a zero of $x_{1} x_{4} x(x-1)^{4} f\left(y_{1}, \ldots, y_{4}\right)$. Hence $\beta^{i_{1}}=\beta^{i_{2}}=\beta^{i_{3}}=\beta^{i_{4}}$ which contradicts the statement. Then, there exists $i \in\{0,1,2,3\}$ such that $i_{5}=i_{1}+i$ fulfills $D_{\left(J \cup\left\{i_{5}\right\}\right)-\left\{i_{j}\right\}}(\beta) \neq 0$ for all $1 \leq j \leq 4$.

We present now two technical results which are needed in Section 5.2 .2

Lemma B.10. Let $(n, m) \in \mathbb{N}^{2}$ with $n>1$ such that $n$ is multiple of $m$ and $m+1$ is multiple of $n-1$. Then $(n, m) \in\{(2,1),(3,1),(2,2),(4,2),(3,3)\}$.

Proof. As $n$ is multiple of $m$ and $m+1$ is multiple of $n-1$, then $m \leq n \leq m+2$. For $m \geq 3$, this inequality implies $n=m$. Thus, if $m \geq 4$, then $(m+1) /(m-1)<2$ and this leads to a contradiction because $m+1$ is a multiple of $m-1$. Analyzing the possible cases for $m=1,2$ we obtain the ordered pairs from the statement.

Theorem B.11. Let $J=\left\{i_{1}, i_{2}, i_{3}, i_{4}\right\}$ be a subset of $\mathcal{A}$. For every root $\beta \in$ $K \backslash\{0,1\}$ of $D_{J}(x)$ there are at least three powers $\left\{\beta^{i_{1}}, \beta^{i_{2}}, \beta^{i_{3}}, \beta^{i_{4}}\right\}$ equal, if and only if $J$ is the affine image $\left(J^{\prime}(\times s)\right)(+r)$ of an exceptional configuration $J^{\prime}$.

Proof. Consider $\beta \neq 0,1$. The set $\left\{\beta^{i_{1}}, \beta^{i_{2}}, \beta^{i_{3}}, \beta^{i_{4}}\right\}$ has three elements equal if $\beta$ is a $s_{j}$-th root of unity for some $j \in\{1,2,3,4\}$, where $s_{1}=\operatorname{gcd}\left(i_{3}-i_{2}, i_{4}-i_{2}\right)$, $s_{2}=\operatorname{gcd}\left(i_{4}-i_{1}, i_{3}-i_{1}\right), s_{3}=\operatorname{gcd}\left(i_{4}-i_{1}, i_{2}-i_{1}\right), s_{4}=\operatorname{gcd}\left(i_{3}-i_{1}, i_{2}-i_{1}\right)$. Note that, if all the powers of $\beta$ are equal, $\beta$ is a $s$-th root of unity where $s=$ $\operatorname{gcd}\left(s_{1}, s_{2}, s_{3}, s_{4}\right)=\operatorname{gcd}\left(i_{4}-i_{1}, i_{3}-i_{1}, i_{2}-i_{1}\right)$. Moreover, note that if $\beta$ is a $s_{j}$-th root of unity for $j \in\{1,2,3,4\}$, then either $\beta^{i_{4}-i_{3}}=1$ or $\beta^{i_{2}-i_{1}}=1$, and both equalities hold if and only if $\beta^{s}=1$.

By Lemma B.4 since every $\beta \neq 0,1$ root of $D_{J}$ satisfy $\beta^{s_{j}}=1$ for some $j \in$ $\{1,2,3,4\}, \beta$ has multiplicity 4 if $\beta^{s}=1$ and multiplicity 1 otherwise. Then, the set of roots of $D_{J}$ (counted with multiplicity) that make at least three $\beta^{i_{j}}$ equal are at most $4 s+\left(i_{4}-i_{3}-s\right)+\left(i_{2}-i_{1}-s\right)=i_{4}-i_{3}+i_{2}-i_{1}+2 s$. Also by Lemma B.2. $D_{J}$ has $i_{4}+i_{3}-i_{2}-i_{1}$ nonzero roots. As every root of $D_{J}$ makes three powers of $\beta$ equal,

$$
i_{4}+i_{3}-i_{2}-i_{1} \leq i_{4}-i_{3}+i_{2}-i_{1}+2 s
$$


or equivalently $i_{3}-i_{2} \leq s$. But as $s$ divides $i_{3}-i_{2}, s=i_{3}-i_{2}$ and therefore $s_{1}=s_{4}=s$. Thus, the only way to have three powers equal and one different is if $\beta$ is a $s_{2}$-th or $s_{3}$-th root of unity, and therefore $i_{4}+i_{3}-i_{2}-i_{1} \leq 4 s+\left(s_{2}-s\right)+\left(s_{3}-s\right)$. As $s_{2} \leq i_{4}-i_{3}, s_{3} \leq i_{2}-i_{1}$ and $2 s=2 i_{3}-2 i_{2}$, so

$$
4 s+\left(s_{2}-s\right)+\left(s_{3}-s\right) \leq i_{4}-i_{3}+i_{2}-i_{1}+2 s=i_{4}+i_{3}-i_{2}-i_{1} .
$$

Hence, all inequalities are, in fact, equalities.

Finally, we analyze the cases when this is possible, that is when $s_{2}=i_{4}-i_{3}$ and $s_{3}=i_{2}-i_{1}$. As $s_{2}=\operatorname{gcd}\left(i_{4}-i_{3}, i_{3}-i_{1}\right)$ and $s_{3}=\operatorname{gcd}\left(i_{4}-i_{2}, i_{2}-i_{1}\right)$, this happens if and only if $i_{3}-i_{1}$ is a multiple of $i_{4}-i_{3}$ and $i_{4}-i_{2}$ is a multiple of $i_{2}-i_{1}$. Considering the integers $n=\frac{i_{3}-i_{1}}{s}, m=\frac{i_{4}-i_{3}}{s}$, then $n$ is a multiple of $m$ and (as $\left.i_{3}-i_{2}=s\right) m+1=\frac{i_{4}-i_{2}}{s}$ is a multiple of $n-1=\frac{i_{2}-i_{1}}{s}$. By Lemma B.10 the possible values for $(n, m)$ are:

- $(n, m)=(2,1)$ in which case $\left(i_{1}, i_{2}, i_{3}, i_{4}\right)=((0,1,2,3)(\times s))\left(+i_{1}\right)$.

- $(n, m)=(3,1)$ in which case $\left(i_{1}, i_{2}, i_{3}, i_{4}\right)=((0,2,3,4)(\times s))\left(+i_{1}\right)$.

- $(n, m)=(2,2)$ in which case $\left(i_{1}, i_{2}, i_{3}, i_{4}\right)=((0,1,2,4)(\times s))\left(+i_{1}\right)$.

- $(n, m)=(4,2)$ in which case $\left(i_{1}, i_{2}, i_{3}, i_{4}\right)=((0,3,4,6)(\times s))\left(+i_{1}\right)$.

- $(n, m)=(3,3)$ in which case $\left(i_{1}, i_{2}, i_{3}, i_{4}\right)=((0,2,3,6)(\times s))\left(+i_{1}\right)$.

As these are exactly the exceptional configurations under affine maps, this completes the proof.

\section{Appendix C. Computations}

We presented several lemmas that require symbolic computations which can be done using mathematical software. We have implemented these computations in Sage [16] and we reproduce here the codes that we have used to check that the claims are correct.

Computation C.1 (For Proposition 4.6).

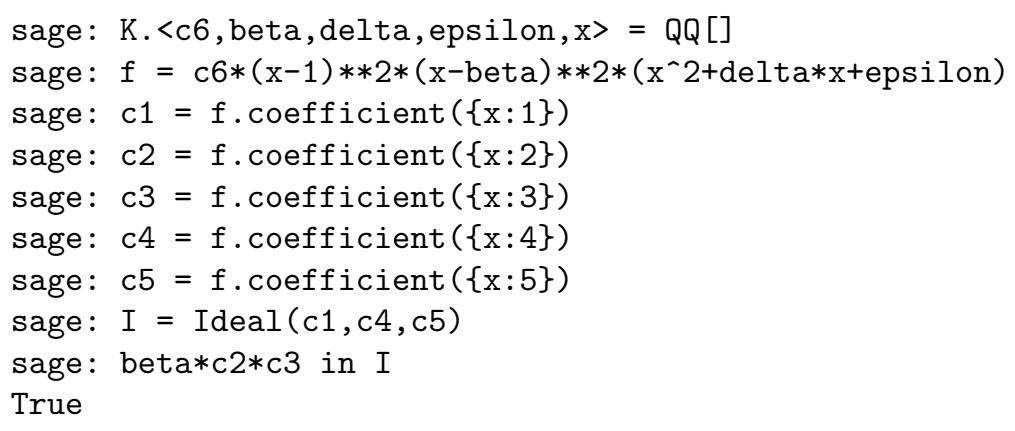

Computation C.2 (For Lemma B.6.

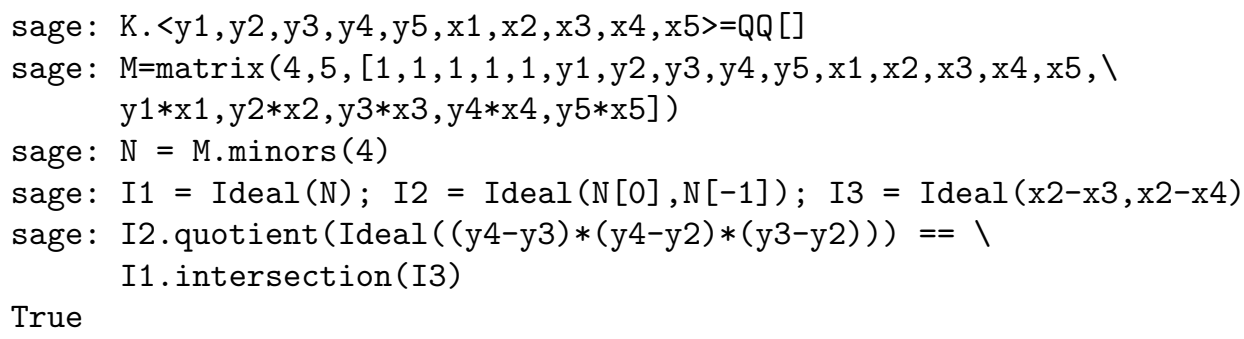

Computation C.3 (For Lemma B.7).

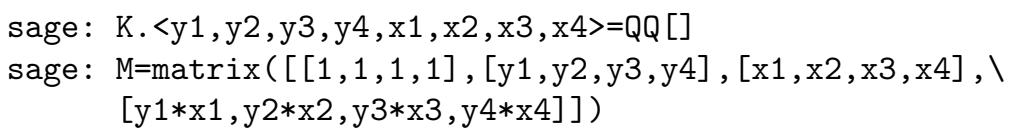


sage: M1=matrix $([[1,1,1,1],[y 1, y 2, y 3, y 4],[x 1, x 2, x 3, x 4], \backslash$

$[\mathrm{y} 1 * \mathrm{y} 1 * \mathrm{x} 1, \mathrm{y} 2 * \mathrm{y} 2 * \mathrm{x} 2, \mathrm{y} 3 * \mathrm{y} 3 * \mathrm{x} 3, \mathrm{y} 4 * \mathrm{y} 4 * \mathrm{x} 4]])$

sage: M21=matrix $([[1,1,1,1],[y 1, y 2, y 3, y 4],[x 1, x 2, x 3, x 4], \backslash$

$[\mathrm{y} 1 * \mathrm{y} 1 *(\mathrm{y} 1-1) * \mathrm{x} 1, \mathrm{y} 2 * \mathrm{y} 2 *(\mathrm{y} 2-1) * \mathrm{x} 2, \mathrm{y} 3 * \mathrm{y} 3 *(\mathrm{y} 3-1) * \mathrm{x} 3, \backslash$ $\mathrm{y} 4 * \mathrm{y} 4 *(\mathrm{y} 4-1) * \mathrm{x} 4]])$

sage: M22=matrix $([[1,1,1,1],[\mathrm{y} 1, \mathrm{y} 2, \mathrm{y} 3, \mathrm{y} 4],[\mathrm{y} 1 * \mathrm{x} 1, \mathrm{y} 2 * \mathrm{x} 2, \mathrm{y} 3 * \mathrm{x} 3, \backslash$ $\mathrm{y} 4 * \mathrm{x} 4],[\mathrm{y} 1 * \mathrm{y} 1 * \mathrm{x} 1, \mathrm{y} 2 * \mathrm{y} 2 * \mathrm{x} 2, \mathrm{y} 3 * \mathrm{y} 3 * \mathrm{x} 3, \mathrm{y} 4 * \mathrm{y} 4 * \mathrm{x} 4]])$

sage: I=Ideal (M.det (), M1 $\cdot \operatorname{det}(), M 21 \cdot \operatorname{det}()+M 22 \cdot \operatorname{det}())$

sage: vandermonde=matrix $([[1,1,1,1],[y 1, y 2, y 3, y 4]$, $[\mathrm{y} 1 * * 2, \mathrm{y} 2 * * 2, \mathrm{y} 3 * * 2, \mathrm{y} 4 * * 2],[\mathrm{y} 1 * * 3, \mathrm{y} 2 * * 3, \mathrm{y} 3 * * 3, \mathrm{y} 4 * * 3]]) \cdot \operatorname{det}() * \mathrm{~K}$

sage: $I 1=I$.quotient (vandermonde)

sage: while I != I1:

$$
\mathrm{I}=\mathrm{I} 1
$$$$
\mathrm{I} 1=\mathrm{I} 1 \text {. quotient (vandermonde) }
$$

sage: I.radical ().quotient (Ideal $(\mathrm{x} 1 * \mathrm{x} 2 * \mathrm{x} 3 * \mathrm{x} 4))$

Ideal ( $x 4-\mathrm{x} 3, \mathrm{x} 4-\mathrm{x} 2, \mathrm{x} 4-\mathrm{x} 1$ ) of Multivariate Polynomial Ring in $\mathrm{y} 1, \mathrm{y} 2, \mathrm{y} 3, \mathrm{y} 4, \mathrm{x} 1, \mathrm{x} 2, \mathrm{x} 3, \mathrm{x} 4$ over Rational Field

Computation C.4 (For Lemma B.8).

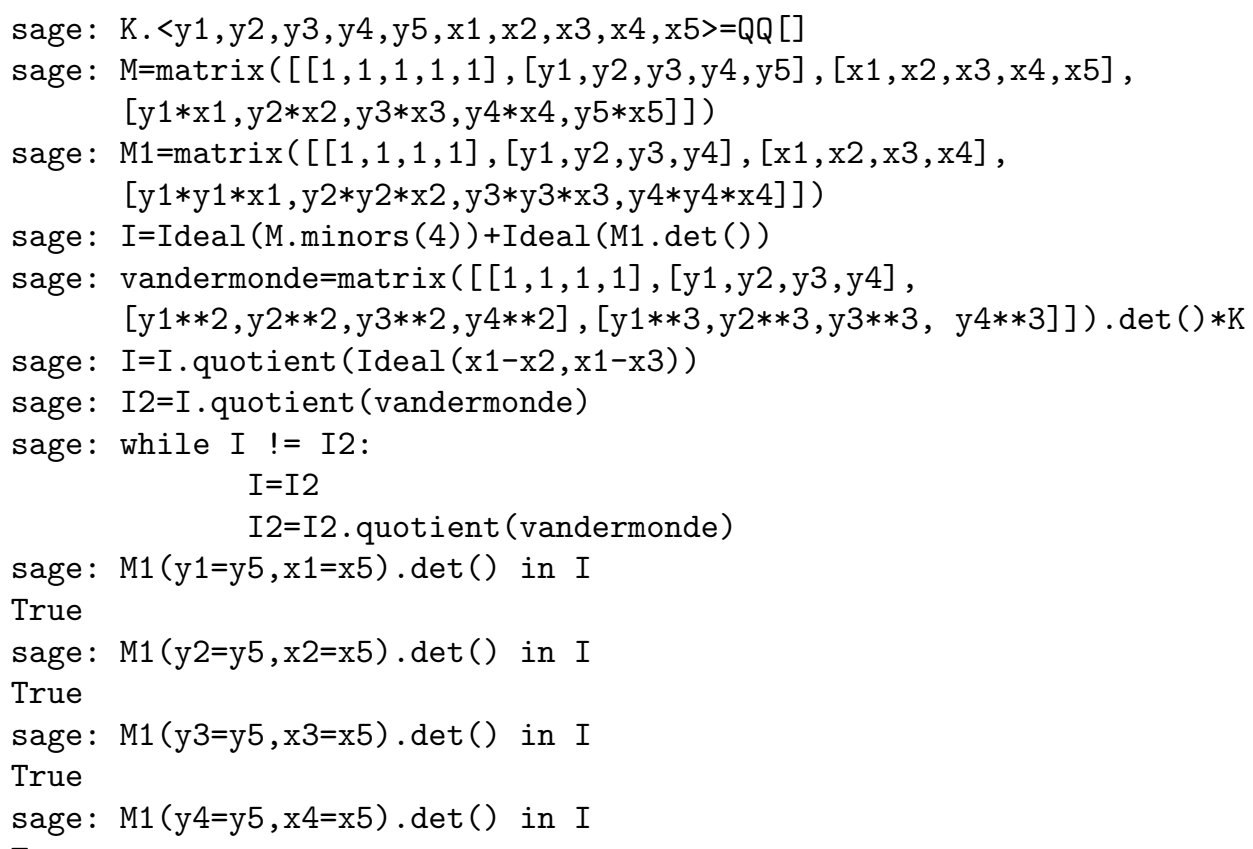

Computation C.5 (For Lemma B.9.

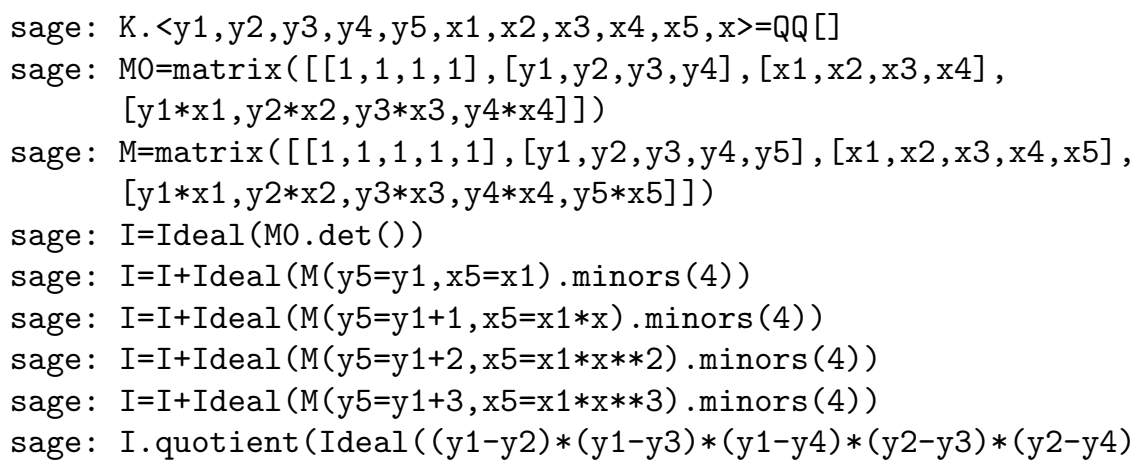


$*(\mathrm{y} 3-\mathrm{y} 4) * \mathrm{x} 1 * \mathrm{x} 4 * \mathrm{x} *(\mathrm{x}-1) * * 4))$

Ideal ( $\mathrm{x} 3-\mathrm{x} 4, \mathrm{x} 2-\mathrm{x} 4, \mathrm{x} 1-\mathrm{x} 4)$ of Multivariate Polynomial Ring

in $\mathrm{y} 1, \mathrm{y} 2, \mathrm{y} 3, \mathrm{y} 4, \mathrm{y} 5, \mathrm{x} 1, \mathrm{x} 2, \mathrm{x} 3, \mathrm{x} 4, \mathrm{x} 5, \mathrm{x}$ over Rational Field

\section{ACKNOWLEDGMENTS}

AD is supported by UBACYT 20020100100242, CONICET PIP 11220110100580 and ANPCyT 2013-1110, Argentina. MIH is supported by UBACyT 20020120100133, CONICET PIP 0099/11 and ANPCyT 2013-1110. She is grateful to the Simons Institute for the Theory of Computing, Berkeley, USA, where this work was developed. LFT is supported by the Spanish Ministerio de Economía y Competitividad and by the European Regional Development Fund (ERDF), under the project MTM201454141-P. We would like to thank Alexander Esterov for useful comments. We are also grateful to Florian Block for his enthusiasm to start this project and for his participation at the early stages of this work.

\section{REFERENCES}

[1] R. Bieri and J.R.J. Groves. The geometry of the set of characters induced by valuations. J. Reine Angew. Math. 347 (1984), 168-195.

[2] F. Block. Counting algebraic curves with tropical geometry. Tropical geometry and integrable systems, Contemp. Math. 580 (2012), 41-54.

[3] T. Bogart, A. N. Jensen, D. Speyer, B. Sturmfels, R. R. Thomas. Computing tropical varieties. J. Symbolic Comput. 42 (2007), no. 1-2, 54-73.

[4] A. Dickenstein, S. di Rocco, and R. Piene. Higher order duality and toric embeddings. Annales de l'institut Fourier, 64 (2014), no. 1, 375-400.

[5] A. Dickenstein, M. Feichtner, and B. Sturmfels. Tropical discriminants. J. Amer. Math. Soc. 20 (2007), 1111-1133.

[6] A. Dickenstein, L. Tabera. Singular tropical hypersurfaces. Discrete Comput. Geom. 47 (2012), no. 2, 430-453.

[7] M. Einsiedler, M. Kapranov, and D. Lind. Non-Archimedean amoebas and tropical varieties. J. Reine Angew. Math. 601 (2006), 139-157.

[8] A. Esterov. Characteristic classes of affine varieties and Plucker formulas for affine morphisms. To appear: Journal of the European Mathematical Society, 2016.

[9] I.M. Gelfand, M.M. Kapranov, and A.V. Zelevinsky. Discriminants, Resultants, and Multidimensional Determinants. Birkhäuser Boston, Boston, MA (1994).

[10] E. Katz. Tropical invariants from the secondary fan. Adv. Geom. 9 (2009), no. 2, $153-180$.

[11] D. Maclagan, B. Sturmfels. Introduction to tropical geometry. Grad. Studies in Math., vol. 161. American Mathematical Society, Providence, RI, 2015.

[12] G. Mikhalkin. Enumerative tropical algebraic geometry in $\mathbb{R}^{2}$. J. Amer. Math. Soc. 18 (2005), no. 2, 313-377.

[13] S. Payne. Fibers of tropicalization. Math. Z. 262 (2009), no. 2, 301-311.

[14] L.F. Tabera. On real tropical bases and real tropical discriminants. Collect. Math. 66 (2015), no. $1,77-92$.

[15] L.F. Tabera. Two Tools in Algebraic Geometry: Construction of Configurations in Tropical Geometry and Hypercircles for the Simplification of Parametric Curves. PhD thesis, Universidad de Cantabria, Université de Rennes I, 2007.

[16] W. A. Stein et al. Sage Mathematics Software (Version 6.9), The Sage Development Team, 2015, htp://www.sagemath.org.

[17] J.J. Yang. Tropical Severi Varieties. Port. Math. 70 (2013), no. 1, 59-91.

[18] J.J. Yang. Secondary fans and Tropical Severi varieties. Manuscripta Math. 149 (2016), no. 1, 93-106.

[19] J. Yu and D. Yuster. Representing tropical linear spaces by circuits. Formal Power Series and Algebraic Combinatorics (FPSAC '07), Proceedings, Tianjin, China, 2007.

A. Dickenstein and M.I. Herrero: Dto. de Matemática, FCEN - Universidad de Buenos Aires, and IMAS (UBA-COniCET), Ciudad Universitaria - Pab. I - C1428EGA Buenos Aires, Argentina

L.F. Tabera: Dto. Matemáticas, Estadística y Computación, Universidad de Cantabria, SPAIN

E-mail address: alidick@dm.uba.ar, iherrero@dm.uba.ar, taberalf@unican.es 